\title{
INDEPENDENT INCREMENT PROCESSES: A MULTILINEARITY PRESERVING PROPERTY
}

\author{
FRED ESPEN BENTH, NILS DETERING AND PAUL KRÜHNER
}

\begin{abstract}
We observe a multilinearity preserving property of conditional expectation for infinite dimensional independent increment processes defined on some abstract Banach space $B$. It is similar in nature to the polynomial preserving property analysed greatly for finite dimensional stochastic processes and thus offers an infinite dimensional generalisation. However, while polynomials are defined using the multiplication operator and as such require a Banach algebra structure, the multilinearity preserving property we prove here holds even for processes defined on a Banach space which is not necessarily a Banach algebra. In the special case of $B$ being a commutative Banach algebra, we show that independent increment processes are polynomial processes in a sense that coincides with a canonical extension of polynomial processes from the finite dimensional case. The assumption of commutativity is shown to be crucial and in a non-commutative Banach algebra the multilinearity concept arises naturally. Some of our results hold beyond independent increment processes and thus shed light on infinite dimensional polynomial processes in general.
\end{abstract}

\section{INTRODUCTION}

An $\mathbb{R}$-valued process $(X(t))_{t \geq 0}$ is said to be a polynomial process if for every polynomial $p$ of degree $n$, there exists another polynomial $q$ of degree at most $n$ such that $\mathbb{E}\left[p(X(t)) \mid \mathcal{F}_{s}\right]=q(X(s))$ for every $t \geq s \geq 0$. The polynomial $q$ may have deterministic time-dependent coefficients. Examples of polynomial processes in $\mathbb{R}$ are affine processes or the multidimensional Jacobi process, among others (see Ackerer, Filipović and Pulido [1] for an application of the Jacobi process to stochastic volatility). Polynomial processes with values in the Euclidean space $\mathbb{R}^{d}, d<\infty$, or subsets thereof have received much attention recently especially due to their applications in financial mathematics. We refer the reader to Cuchiero, Keller-Ressel and Teichmann [9], Filipović and Larsson [13] and Foreman and Sørensen [14, and the references therein for an analysis and application of these processes and an overview of the existing literature.

In the present paper we lift the notion of polynomial processes to general Banach spaces with a particular focus on independent increment processes. We thus show in a first instance how polynomial processes can be extended to an infinite dimensional setting and shed light on the special role commutative Banach algebras play in this

Date: March 24, 2021.

Key words and phrases. Infinite dimensional stochastic processes, polynomial processes, Banach algebras, conditional expectation, multilinear maps.

FEB acknowledges financial support from FINEWSTOCH, a research project funded by the Norwegian Research Council. We are grateful to the referee and associate editor for suggestions that greatly improved the presentation of the paper. 
context. We introduce a multilinearity preserving property for processes in a general Banach space and a polynomial preserving property for processes with values in a Banach algebra.

To explain our approach in slightly more detail, let $\mathcal{L}_{n}: B^{n} \rightarrow B, n \in \mathbb{N}$, be a multilinear map on $B^{n}$, the product space of $n$ copies of the Banach space $B$. We say that a $B$-valued stochastic process $(X(t))_{t \geq 0}$ is a multilinear process if for every $n \in \mathbb{N}$ and every multilinear map $\mathcal{L}_{n}$, it holds that

$$
\mathbb{E}\left[\mathcal{L}_{n}(X(t), \ldots, X(t)) \mid \mathcal{F}_{s}\right]=\sum_{k=0}^{n} \mathcal{M}_{k}(X(s ; t), \ldots, X(s ; t))
$$

for all $t \geq s \geq 0$. Here, for $1 \leq k \leq n, \mathcal{M}_{k}$ are again multilinear maps, now on $B^{k}$ and $\mathcal{M}_{0} \in B$ is a constant. Further $X(s ; t)$ is an $\mathcal{F}_{s}$-measurable random variable with values in $B$. Often this is simply $X(s)$, and moreover, the multilinear maps $\mathcal{M}_{k}$ may be depending (deterministically) on $t$ and $s$. This of course includes the representation for monomials (and by linearity also polynomials) by defining $\mathcal{L}_{n}: B^{n} \rightarrow B$ by $\mathcal{L}_{n}\left(x_{1}, \ldots, x_{n}\right)=x_{1} \cdots x_{n}$ if a designated multiplication in $B$ is defined. The multilinearity property thus extends naturally the idea of the polynomial property in that moment like quantities of $(X(t))_{t \geq 0}$ can be easily calculated. The structure, however, does not focus on the particular moments arising from the designated multiplication operator.

Our first main Theorem 8 shows that independent increment processes (and variants thereof) in a Banach space are multilinearity preserving processes. If the Banach space has a multiplication defined and forms a Banach algebra, we show in Theorem 16 and Proposition 18 that they are also (generalized) polynomial processes. Moreover, in Proposition 13 we show that in most cases, the multilinear preserving property allows to calculate conditional expectations even of multilinear forms in contrast to maps, a property crucial for applications. As auxiliary results we derive several specific properties of conditional expectations in (possibly noncommutative) Banach algebras, which might be of independent interest.

Applications: There is a range of possible applications for our results. We work out two of them in more detail. First, we show how the multilinearity property comes in handy to calculate conditional moments of the norm of the process. The efficient calculation of moments is important for instance in order to determine population parameters in statistical estimation. Second, we apply our results to the pricing of options on commodity forwards when the entire forward curve is modeled as an element in the Filipović space (see for instance Benth and Krühner [4]). In this setting a commutative Banach algebra can be defined by pointwise multiplication of the forward prices and we exploit the generated polynomial structure for pricing options on forwards. This leads to an easy to calculate formula for pricing European options on the forward price.

Additionally we show how processes whose values are measures can be treated, linking our analysis and definitions to the work of Cuchiero, Larsson and SvalutoFerro [10]. Examples of relevance for non-commutative Banach algebras include matrix-valued stochastic processes or more general processes of linear bounded operators where multiplication is the concatenation of operators. These cases cover infinite-dimensional stochastic volatility models (see Benth, Rüdiger and Süss [5]).

The outline of the paper is as follows. In Section 2 we introduce our notion of a multilinear process and prove our first main results for processes with values in a 
Banach space. In Section 3 we restrict the state space to be a commutative Banach algebra and analyse polynomial versus multilinear processes. We pay special attention to the Ornstein-Uhlenbeck dynamics. Finally, in Section 4 we provide several possible applications of our results. The Appendix A contains some important auxiliary results about conditional expectation in Banach spaces and algebras, which we could not find in the literature.

\section{Multilinear maps And multilinear PROCESSES}

In this section we study stochastic processes with values in a Banach space $B$ which possess certain stability properties with respect to "polynomials" and conditional expectation. We introduce polynomials via certain multilinear maps, that are defined next:

Denote by $B^{k}=B \times \cdots \times B$ the product space of $k \in \mathbb{N}$ copies of $B$ equipped with the norm $\|\cdot\|_{k}:=\sup _{1 \leq i \leq k}\|\cdot\|$. The product space $B^{k}$ becomes again a Banach space. We introduce the following definition of $k$-linear maps, that will play an important role in the sequel:

Definition 1. We say that $\mathcal{L}_{k}: B^{k} \rightarrow B$ for $k \in \mathbb{N}$ is a $k$-linear map if it is linear in each argument in the sense that for any $x_{1}, x_{2}, \ldots, x_{k}, y \in B$ and $a, b \in \mathbb{F}$

$$
\begin{aligned}
\mathcal{L}_{k}\left(x_{1}, \ldots, x_{j-1}, a x_{j}+b y, x_{j+1}, \ldots, x_{k}\right) \\
\quad=a \mathcal{L}_{k}\left(x_{1}, \ldots, x_{k}\right)+b \mathcal{L}_{k}\left(x_{1}, \ldots, x_{j-1}, y, x_{j+1}, \ldots, x_{k}\right)
\end{aligned}
$$

for each $j=1, \ldots, k$. A $k$-linear map $\mathcal{L}_{k}$ is bounded if there exists a constant $K>0$ such that

$$
\left\|\mathcal{L}_{k}\left(x_{1}, \ldots, x_{k}\right)\right\| \leq K\left\|x_{1}\right\| \cdots\left\|x_{k}\right\|
$$

for all $x_{1}, \ldots, x_{k} \in B$. We denote the space of bounded $k$-linear maps by $L_{k}(B)$.

Notice that $L_{1}(B)=L(B)$, the space of bounded linear operators. Often we will call a $k$-linear map simply multilinear without specifying the dimension.

A $k$-linear map $\mathcal{L}_{k}$ induces a $k$-monomial $\mathcal{M}_{k}: B \rightarrow B$ by

$$
\mathcal{M}_{k}(x):=\mathcal{L}_{k}(x, \ldots, x) .
$$

If $\mathcal{L}_{k} \in L_{k}(B)$, we see that $\left\|\mathcal{M}_{k}(x)\right\| \leq K\|x\|^{k}$, and we denote the set of all such $k$ monomials by $M_{k}(B)$. Of course, $M_{1}(B)=L(B)$, the space of bounded operators. Additionally, we define $M_{0}(B):=B$ for completeness. $M_{0}(B)$ will play the role as the space of "constants", or, zero-order monomials. We remark that $M_{k}(B)$ is a vector space over the same field as $B$. We have the following result showing that the monomials are locally Lipschitz continuous on $B$ :

Proposition 2. If $\mathcal{M}_{k} \in M_{k}(B)$, then for any $x, y \in B$

$$
\left\|\mathcal{M}_{k}(x)-\mathcal{M}_{k}(y)\right\| \leq C(\|x\|,\|y\|)\|x-y\|
$$

where $C(\|x\|,\|y\|)=K \sum_{i=1}^{k}\|x\|^{k-i}\|y\|^{i-1}$ for some positive constant $K$.

Proof. We notice that for $k=1, \mathcal{M}_{1} \in L(B)$ and therefore Lipschitz continuous. Let therefore $k \geq 2$. As $\mathcal{M}_{k} \in M_{k}(B)$, we have for $x \in B$ that $\mathcal{M}_{k}(x)=$ $\mathcal{L}_{k}(x, \ldots, x)$ for a bounded $k$-linear map, $\mathcal{L}_{k} \in L_{k}(B)$. By adding and subtracting $\mathcal{L}_{k}(y, \ldots, y, x, \ldots, x)$, where $y \in B$ goes successively through all the $k-1$ first 
coordinates, we find from the triangle inequality and the multilinearity property of $\mathcal{L}_{k}$,

$$
\begin{aligned}
\left\|\mathcal{M}_{k}(x)-\mathcal{M}_{k}(y)\right\|=\| & \mathcal{L}_{k}(x, \ldots, x)-\mathcal{L}_{k}(y, \ldots, y) \| \\
\leq \| & \left\|\mathcal{L}_{k}(x, \ldots, x)-\mathcal{L}_{k}(y, x, \ldots, x)\right\| \\
& +\left\|\mathcal{L}_{k}(y, x, \ldots, x)-\mathcal{L}_{k}(y, y, x, \ldots, x)\right\| \\
& +\cdots \\
& +\left\|\mathcal{L}_{k}(y, \ldots, y, x)-\mathcal{L}_{k}(y, \ldots, y)\right\| \\
=\| & \mathcal{L}_{k}(x-y, \ldots, x) \| \\
& +\left\|\mathcal{L}_{k}(y, x-y, \ldots, x)\right\| \\
& +\cdots \\
& +\left\|\mathcal{L}_{k}(y, \ldots, y, x-y)\right\| \\
\leq & K\|x-y\|\|x\|^{k-1}+K\|y\|\|x-y\|\|x\|^{k-2}+\ldots \\
& +K\|y\|^{k-1}\|x-y\| .
\end{aligned}
$$

The last inequality follows from the boundedness of $\mathcal{L}_{k}$. The result follows.

Let $(X(t))_{t \geq 0}$ be a $B$-valued stochastic process, that is, a family of $B$-valued random variables $X(t)$ indexed by $t \geq 0$. In the following we shall be interested in the conditional expectation $\mathcal{M}_{k}(X(t))$ given $\mathcal{F}_{s}$ for $t \geq s \geq 0$ where $\mathcal{M}_{k} \in M_{k}(B)$. More specifically, we want to define and study processes $(X(t))_{t \geq 0}$ where for any $\mathcal{M}_{k} \in M_{k}(B)$ there exists a family of $j$ th-order monomials $\overline{\mathcal{M}}_{j} \in M_{j}(B)$ with $j \leq k$ such that

$$
\mathbb{E}\left[\mathcal{M}_{k}(X(t)) \mid \mathcal{F}_{s}\right]=\sum_{j=0}^{k} \overline{\mathcal{M}}_{j}(X(s ; t)),
$$

and where $X(s ; t)$ is some strongly $\mathcal{F}_{s}$-measurable random variable. As we see, we are interested in processes which preserve the "polynomial" order, as the monomials on the right hand side are not exceeding $k$ in their orders. Moreover, the $j$ th-order monomials $\overline{\mathcal{M}}_{j}$ are allowed to depend (deterministically) on $s$ and $t$, however, we do not state this explicitly to lessen the notational burden.

A minimal requirement for studying (2) is that $\mathcal{M}_{k}(X(t))$ is Bochner integrable. As $X(t)$ is strongly measurable and $\mathcal{M}_{k}$ is continuous by Proposition 2 , it follows from Lemma 21 in Appendix A that $\mathcal{M}_{k}(X(t))$ is strongly measurable. We introduce the following assumption:

Assumption 3. The process $(X(t))_{t>0}$ has finite moments of all order, i.e., for any $n \in \mathbb{N}, \mathbb{E}\left[\|X(t)\|^{n}\right]<\infty$ for all $t \geq 0$.

Since we have $\left\|\mathcal{M}_{k}(x)\right\| \leq K\|x\|^{k}$, it follows under Assumption 3 that $\mathcal{M}_{k}(X(t))$ is Bochner integrable, and in particular the conditional expectation in (2) exists. In the study of polynomial diffusions in $\mathbb{R}^{k}$, a moment assumption is usually not needed and follows for polynomial processes from $\mathbb{E}\left[\|X(0)\|^{n}\right]<\infty$ for all $n$ (see Thm. 3.1. in Filipović and Larsson [13]). For general state space and driving noise however, we will need it.

As a simple example, let us look at the case $B=\mathbb{R}$ and the function $\mathcal{M}_{k}: \mathbb{R} \rightarrow \mathbb{R}$ given by $\mathcal{M}_{k}(x)=x^{k}$. Then one easily observes that $\mathcal{M}_{k}$ is induced by the $k$ linear map $\mathcal{L}_{k}: \mathbb{R} \times \cdots \times \mathbb{R} \rightarrow \mathbb{R},\left(x_{1}, \ldots, x_{k}\right) \rightarrow x_{1} \cdots x_{k}$. In Cuchiero et 
al. 9] and Filipović and Larsson [13, a real-valued $\mathcal{F}_{t}$-adapted stochastic process $(X(t))_{t \geq 0}$ is called a polynomial process if for any $n \in \mathbb{N}, \mathbb{E}\left[(X(t))^{n} \mid \mathcal{F}_{s}\right]=q_{n}(X(s))$ for some polynomial $q_{n}$ of degree at most $n$. We will later see that $k$-linear maps arise naturally when dealing with polynomials in possibly non-commutative Banach algebras.

Next, let us define multilinear processes:

Definition 4. Let $(X(t))_{t \geq 0}$ be a B-valued stochastic process and $(X(s ; t))_{0 \leq s \leq t<\infty}$ a family of $B$-valued random variables, such that $X(s ; t)$ is strongly $\mathcal{F}_{s}$-measurable. The process $(X(t))_{t \geq 0}$ is said to be a multilinear process with respect to the family $(X(s ; t))_{0 \leq s \leq t<\infty}$ if for any $k \in \mathbb{N}$ and $\mathcal{M}_{k} \in M_{k}(B)$, there exists a family of $j$ th-order monomials $\overline{\mathcal{M}}_{j} \in M_{j}(B), j \leq k$, such that for all $s \leq t$ it holds,

$$
\mathbb{E}\left[\mathcal{M}_{k}(X(t)) \mid \mathcal{F}_{s}\right]=\sum_{j=0}^{k} \overline{\mathcal{M}}_{j}(X(s ; t)) .
$$

Note that if we take a linear combination of monomials up to order $k \in \mathbb{N}$, that is, $\mathcal{P}_{k}:=\sum_{j=0}^{k} p_{j} \mathcal{M}_{j}$ for $p_{j} \in \mathbb{F}$ and $\mathcal{M}_{j} \in M_{j}(B)$ for $j=0, \ldots, k$, we find by the vector space structure of $M_{j}(B)$ that $\mathcal{P}_{k}$ can be represented by a sum of monomials up to degree $k$. Hence, we can use a linear combination of monomials in the conditional expectation defining a multilinear process in Definition 4.

Remark 5. Instead of considering $\mathbb{E}\left[\mathcal{M}_{k}(X(t)) \mid \mathcal{F}_{s}\right]$ with $\mathcal{M}_{k} \in M_{k}(B)$ one could consider a $k$-linear form $\mathcal{L}_{k}: B^{k} \rightarrow \mathbb{F}$ and their expectation $\mathbb{E}\left[\mathcal{M}_{k}(X(t)) \mid \mathcal{F}_{s}\right]$. We show in Proposition 13 that when $B$ is a Hilbert space and the process $(X(t))_{t \geq 0}$ is multilinear, then the representation of $k$-linear forms follows for multilinear processes. Therefore the multilinearity property is stronger and we consider it here. Moreover, if a dedicated multiplication operator exists then it is a bilinear form and the notion of polynomials is more naturally extended in this setting.

We notice that in Definition 4 we claim the existence of a family of $\mathcal{F}_{s}$-measurable random variables $(X(s ; t))_{0 \leq s \leq t<\infty}$, rather than using $X(s)$ as argument on the right-hand side in (3). As we will see in Example 7 this allows us to show that mild solutions to certain stochastic partial differential equations as the OrnsteinUhlenbeck process are polynomial processes with respect to a smart choice of $(X(s ; t))_{0 \leq s \leq t<\infty}$. To capture these processes also our following definition of independent increment processes is a rather generous one and even for the case $B=\mathbb{R}$ includes processes which are not independent increment processes in the conventional sense. We define independent increment processes on general Banach spaces as:

Definition 6. The process $(X(t))_{t \geq 0}$ is called an independent increment process if

(1) $X(t)$ is strongly $\mathcal{F}_{t}$-measurable for any $t \geq 0$,

(2) for every $t$ and every $s \leq t$, there exists a decomposition of $X(t)$ into a strongly $\mathcal{F}_{s}$-measurable part $X^{\|}(s ; t)$ and a part $X^{\perp}(s ; t)$ that is independent of $\mathcal{F}_{s}$ such that $X(t)=X^{\perp}(s ; t)+X^{\|}(s ; t)$,

(3) all moments of $\left\|X^{\perp}(s ; t)\right\|$ and $\left\|X^{\|}(s ; t)\right\|$ are integrable.

Applebaum [3] defines a Lévy process on a separable Banach space as a $B$-valued stochastically continuous process $(L(t))_{t \geq 0}$ which is $\mathcal{F}_{t}$-adapted, the increments 
$L(t)-L(s)$ are independent of $\mathcal{F}_{s}$ for any $t>s \geq 0$ with distribution only depending on $t-s$, and having càdlàg paths. In view of Definition 6. $(L(t))_{t \geq 0}$ will be an independent increment process with $L^{\perp}(s ; t):=L(t)-L(s)$ and $L^{\|}(s ; t):=L(s)$ as long as all moments of $\|L(t)\|$ are integrable for any $t \geq 0$. Property (3) in Definition 6 follows by the fact that $L(t)-L(s) \stackrel{d}{=} L(t-s)$ by definition of the Lévy process. The canonical example of a Lévy process is the Wiener process. In a separable Banach space, Fernique's Theorem (see Peszat and Zabczyk [17]) ensures the moment condition (3) in Definition 6 for a Wiener process. Also note that Property (3) in Definition 6 implies especially that $\mathbb{E}\left[\|X(t)\|^{n}\right]<\infty$ for all $n \in \mathbb{N}$. We now provide a more interesting example of an independent increment process according to Definition 6 .

Example 7. Let $\left(\mathcal{S}_{t}\right)_{t \geq 0}$ be a $C_{0}$-semigroup on $B$ and $(W(t))_{t \geq 0}$ a $B$-valued Wiener process. By Fernique's Theorem all moments of $\|W(t)\|$ are finite. Consider the stochastic process $(X(t))_{t \geq 0}$ given by

$$
X(t)=\int_{0}^{t} \mathcal{S}_{t-s} d W(s) .
$$

As $(W(t))_{t \geq 0}$ in particular is square-integrable, it follows that the stochastic convolution $(X(t))_{t \geq 0}$ is a well-defined $\mathcal{F}_{t}$-adapted process in $B$ (see Applebaum [3] and Peszat and Zabczyk [17]). Moreover, it is known (see again Applebaum [3] and Da Prato and Zabczyk [18]) that $(X(t))_{t \geq 0}$ is a mild solution of the stochastic evolution equation

$$
d X(t)=\mathcal{A} X(t) d t+d W(t)
$$

where $\mathcal{A}$ is the (densely defined) generator of $\left(\mathcal{S}_{t}\right)_{t \geq 0}$. Furthermore, $(X(t))_{t \geq 0}$ is a symmetric Gaussian process and by Fernique's Theorem all moments of $\|X(t)\|$ are finite.

We decompose $X(t)$ in (4) into $X^{\perp}(s ; t):=\int_{s}^{t} \mathcal{S}_{t-u} d W(u)$ and $X^{\|}(s ; t):=$ $\int_{0}^{s} \mathcal{S}_{t-u} d W(u)$. We find that $X^{\perp}(s ; t)$ is independent of $\mathcal{F}_{s}$ and $X^{\|}(s ; t)$ is $\mathcal{F}_{s^{-}}$ measurable and thus $(X(t))_{t \geq 0}$ is an independent increment process. Processes of the form (4) are relevant in financial mathematics. We provide an example from commodity markets later in the application section, Sect. 4.

Theorem 8. Suppose that $(X(t))_{t \geq 0}$ is an independent increment process and let $\mathcal{M}_{k} \in M_{k}(B)$. Then there exists a family of $j$ th-order monomials $\overline{\mathcal{M}}_{j} \in M_{j}(B)$, $0 \leq j \leq k$, such that

$$
\mathbb{E}\left[\mathcal{M}_{k}(X(t)) \mid \mathcal{F}_{s}\right]=\sum_{j=0}^{k} \overline{\mathcal{M}}_{j}\left(X^{\|}(s ; t)\right),
$$

for any $s \leq t$, where the $\overline{\mathcal{M}}_{j}$ 's depend on $s$ and $t$. In other words, $(X(t))_{t \geq 0}$ is a multilinear process with respect to $\left(X^{\|}(s ; t)\right)_{0 \leq s \leq t<\infty}$.

Proof. For $k=0$ the claim is trivial, so assume that $k \geq 1$. Let $\mathcal{L}_{k} \in L_{k}(B)$ be such that $\mathcal{M}_{k}(v)=\mathcal{L}_{k}(v, \ldots, v)$. Recall by Definition 6 that $X(t)=X^{\perp}(s ; t)+X^{\|}(s ; t)$ for all $0 \leq s \leq t<\infty$, where $X^{\|}(s ; t)$ is strongly $\mathcal{F}_{s}$-measurable and $X^{\perp}(s ; t)$ is 
independent of $\mathcal{F}_{s}$. Thus we get from multilinearity of $\mathcal{L}_{k}$

$$
\begin{aligned}
\mathcal{M}_{k}(X(t))= & \mathcal{L}_{k}(X(t), \ldots, X(t)) \\
= & \mathcal{L}_{k}\left(X^{\perp}(s ; t)+X^{\|}(s ; t), \ldots, X^{\perp}(s ; t)+X^{\|}(s ; t)\right) \\
= & \mathcal{L}_{k}\left(X^{\|}(s ; t), X^{\perp}(s ; t)+X^{\|}(s ; t), \ldots, X^{\perp}(s ; t)+X^{\|}(s ; t)\right) \\
& \quad+\mathcal{L}_{k}\left(X^{\perp}(s ; t), X^{\perp}(s ; t)+X^{\|}(s ; t), \ldots, X^{\perp}(s ; t)+X^{\|}(s ; t)\right) .
\end{aligned}
$$

Continuing like this over the remaining $k-1$ arguments one can decompose the above expression into a linear combination of $2^{k}$ terms of the form

$$
\mathcal{L}_{k}\left(Y_{j, 1}, \ldots, Y_{j, k}\right)
$$

for $1 \leq j \leq 2^{k}$ with $Y_{j, i} \in\left\{X^{\perp}(s ; t), X^{\|}(s ; t)\right\}$, and there are exactly $\left(\begin{array}{l}k \\ n\end{array}\right)$ terms $j$ for which $\#\left\{Y_{j, i} \mid Y_{j, i}=X^{\|}(s ; t)\right\}=n$.

Let us look at a particular term where $X^{\|}(s ; t)$ appears in the first two arguments. Introduce the function $\mathcal{L}_{2,1}: B \times B \rightarrow B$ defined as

$$
\mathcal{L}_{2,1}\left(y_{1}, y_{2}\right)=\mathbb{E}\left[\mathcal{L}_{k}\left(y_{1}, y_{2}, X^{\perp}(s ; t), \ldots, X^{\perp}(s ; t)\right)\right] .
$$

The subscript $(2,1)$ denotes that $\mathcal{L}_{2,1}$ is the function related to the first term in which $X^{\|}(s ; t)$ appears twice, where the ordering is irrelevant. In view of Proposition 23 in Appendix A, let $f(x, y)=\mathcal{L}_{k}(y, y, x, \ldots, x), X=X^{\perp}(s ; t)$ and $Y=X^{\|}(s ; t)$. Then, $\sigma(X)=\sigma\left(X^{\perp}(s ; t)\right)$ and $\mathcal{F}_{s}$ are independent, and $Y=X^{\|}(s ; t)$ is strongly $\mathcal{F}_{s}$-measurable. First, we show that $(x, y) \mapsto f(x, y)$ is continuous: Indeed, for $(x, y),(u, v) \in B \times B$, we find by triangle inequality and $\mathcal{L}_{k} \in L_{k}(B)$ that

$$
\begin{aligned}
\|f(u, v)-f(x, y)\|=\| & \mathcal{L}_{k}(v, v, u, \ldots, u)-\mathcal{L}_{k}(y, y, x, \ldots, x) \| \\
\leq \| & \mathcal{L}_{k}(v, v, u, \ldots, u)-\mathcal{L}_{k}(y, v, u, \ldots, u) \| \\
& +\left\|\mathcal{L}_{k}(y, v, u, \ldots, u)-\mathcal{L}_{k}(y, y, u, \ldots, u)\right\| \\
& +\left\|\mathcal{L}_{k}(y, y, u, \ldots, u)-\mathcal{L}_{k}(y, y, x, u, \ldots, u)\right\| \\
& \quad+ \\
& +\left\|\mathcal{L}_{k}(y, y, x, \ldots, x, u)-\mathcal{L}_{k}(y, y, x, \ldots, x)\right\| \\
=\| & \mathcal{L}_{k}(v-y, v, u, \ldots, u)\|+\| \mathcal{L}_{k}(y, v-y, u, \ldots, u) \| \\
& +\left\|\mathcal{L}_{k}(y, y, u-x, u, \ldots, u)\right\| \\
& +\cdots \\
& +\left\|\mathcal{L}_{k}(y, y, x, \ldots, x, u-x)\right\| \\
\leq K & \|v-y\|\|v\|\|u\|^{k-2}+K\|y\|\|v-y\|\|u\|^{k-2} \\
& +K\|y\|^{2}\|u-x\|\|u\|^{k-3}+\cdots+K\|y\|^{2}\|u-x\|\|x\|^{k-2} \\
=K & \|u\|^{k-2}(\|v\|+\|y\|)\|v-y\| \\
& +K\|y\|^{2}\left(\sum_{n=0}^{k-3}\|x\|^{n}\|u\|^{k-3-n}\right)\|u-x\|
\end{aligned}
$$

Thus, $(x, y) \mapsto f(x, y)$ is a local Lipschitz continuous map from $B \times B$ into $B$. 
Continuity implies that $f(X, y)$ is strongly measurable (see Lemma 21 in Appendix A. Since

$$
\|f(X, y)\| \leq K\|y\|^{2}\|X\|^{n-2}
$$

it follows that $f(X, y)$ is Bochner integrable by the finite moments condition on $\|X\|=\left\|W^{\perp}(s ; t)\right\|$. Furthermore,

$$
\| f(X, \tilde{Y})]\|\leq K\| \tilde{Y}\left\|^{2}\right\| X\left\|^{k-2} \leq K\right\| Y\left\|^{2}\right\| X \|^{k-2}=: \tilde{Z}
$$

provided that $\|\tilde{Y}\| \leq\|Y\|$ and therefore the bound in (30) holds. Again using (7) it follows that $\|\mathbb{E}[f(X, y)]\| \leq K\|y\|^{2} \mathbb{E}\left[\|X\|^{k-2}\right]$ and

$$
\left\|\mathbb{E}[f(X, y)]_{y=\tilde{Y}}\right\| \leq K\|\tilde{Y}\|^{2} \mathbb{E}\left[\|X\|^{k-2}\right] \leq K\|Y\|^{2} \mathbb{E}\left[\|X\|^{k-2}\right]=: Z
$$

provided that $\|\tilde{Y}\| \leq\|Y\|$ and the bound in 29 holds. Moreover, appealing to the fact that $\mathcal{L}_{k} \in L_{k}(B)$ and Bochner's inequality together with the finiteness of all moments of $\|X\|$, we find by using similar arguments as above that $y \mapsto \mathbb{E}[f(X, y)]$ is locally Lipschitz continuous. Thus we can apply Proposition 23 in Appendix A and conclude that

$$
\begin{aligned}
\mathcal{L}_{2,1}\left(X^{\|}(s ; t), X^{\|}(s ; t)\right) & =\mathbb{E}\left[\mathcal{L}_{k}\left(y, y, X^{\perp}(s ; t), \ldots, X^{\perp}(s ; t)\right)\right]_{y=X \|}(s ; t) \\
& =\mathbb{E}\left[\mathcal{L}_{k}\left(X^{\|}(s ; t), X^{\|}(s ; t), X^{\perp}(s ; t), \ldots, X^{\perp}(s ; t)\right) \mid \mathcal{F}_{s}\right] .
\end{aligned}
$$

By linearity of the expectation operator along with multilinearity of $\mathcal{L}_{k}$, the function $\mathcal{L}_{2,1}\left(y_{1}, y_{2}\right)$ is bilinear and indeed an element of $L_{2}(B)$. The same argument applies to the other $\left(\begin{array}{l}k \\ 2\end{array}\right)-1$ terms $\mathcal{L}_{2,2}, \ldots, \mathcal{L}_{2,\left(\begin{array}{c}k \\ 2\end{array}\right)}$ with $X^{\|}(s ; t)$ appearing twice. Since the sum of bilinear maps is bilinear we can define the bilinear function:

$$
\widetilde{\mathcal{L}}_{2}\left(y_{1}, y_{2}\right)=\sum_{i=1}^{\left(\begin{array}{c}
k \\
2
\end{array}\right)} \mathcal{L}_{2, i}\left(y_{1}, y_{2}\right)
$$

and $\overline{\mathcal{M}}_{2}(y):=\widetilde{\mathcal{L}}_{2}(y, y) \in M_{2}(B)$. In the same way the other functions $\overline{\mathcal{A}}_{j}\left(X^{\|}(s ; t)\right)$ can be defined for $j \in\{1,3, \ldots, k\}$ and the representation (6) follows. Thus, the theorem is proved.

We next observe that elements of $M_{k}(B)$ share similar characteristics as the monomials on the real line. In fact their $(k+1)$-th Fréchet derivative vanishes.

Proposition 9. Assume for $k \in \mathbb{N}$ that $\mathcal{M}_{k} \in M_{k}(B)$ is induced by $\mathcal{L}_{k} \in L_{k}(B)$. Then the $n$-th Fréchet derivative $D^{n} \mathcal{M}_{k}: B \rightarrow L_{n}(B)$ is given by

$$
D^{n} \mathcal{M}_{k}(u)\left(h_{1}, \ldots, h_{n}\right)=\sum_{\substack{x_{i} \in\left\{u, h_{1}, \ldots, h_{n}\right\} \\ \#\left\{i \mid x_{i}=h_{j}\right\}=1 \\ 1 \leq j \leq n}} \mathcal{L}_{k}\left(x_{1}, \ldots, x_{k}\right)
$$

for $n=1, \ldots, k$ and $D^{k+1} \mathcal{M}_{k}(u)\left(h_{1}, \ldots, h_{k+1}\right)=0$.

Proof. We have $\mathcal{M}_{k}(u)=\mathcal{L}_{k}(u, \ldots, u)$. Using the chain rule for the Fréchet derivative we then get that

$$
D \mathcal{M}_{k}(u)=\sum_{i=1}^{k} \nabla_{i} \mathcal{L}_{k}(u, \ldots, u) \cdot 1
$$


To calculate $\nabla_{i} \mathcal{L}_{k}(u, \ldots, u)$, observe that since $\mathcal{L}_{k}\left(u+h_{1}, \ldots, u\right)-\mathcal{L}_{k}(u, \ldots, u)-$ $\mathcal{L}_{k}\left(h_{1}, \ldots, u\right)=0$ by multilinearity and therefore by the definition of the Fréchet derivative

$$
\lim _{\left\|h_{1}\right\| \rightarrow 0} \frac{\left\|\mathcal{L}_{k}\left(u+h_{1}, u, \ldots, u\right)-\mathcal{L}_{k}(u, u, \ldots, u)-\mathcal{L}_{k}\left(h_{1}, u, \ldots, u\right)\right\|}{\left\|h_{1}\right\|}=0 .
$$

Hence, $\nabla_{1} \mathcal{L}_{k}(u, \ldots, u)\left(h_{1}\right)=\mathcal{L}_{k}\left(h_{1}, u, \ldots, u\right)$, and more generally $\nabla_{i} \mathcal{L}_{k}(u, \ldots, u)\left(h_{1}\right)=$ $\mathcal{L}_{k}\left(u, \ldots, u, h_{1}, u, \ldots, u\right)$, where the entry $h_{1}$ is in the $i$-th coordinate. It follows that,

$$
D \mathcal{M}_{k}(u)\left(h_{1}\right)=\sum_{\substack{x_{i} \in\left\{u, h_{1}\right\} \\ \#\left\{i \mid x_{i}=h_{1}\right\}=1}} \mathcal{L}_{k}\left(x_{1}, \ldots, x_{k}\right) .
$$

Clearly $D \mathcal{M}_{k}$ maps from $B$ to $L_{1}(B)=L(B)$.

The claim now follows by induction: assume that $(9)$ holds for $n<k$. We pick the term $\mathcal{L}_{k}\left(u, \ldots, u, h_{1}, \ldots, h_{n}\right)$ from the sum in $(9)$. Then

$$
\begin{aligned}
D \mathcal{L}_{k}\left(u, \ldots, u, h_{1}, \ldots, h_{n}\right)\left(h_{n+1}\right)= & \sum_{i=1}^{k-n} \nabla_{i} \mathcal{L}_{k}\left(u, \ldots, u, h_{1}, \ldots, h_{n}\right)\left(h_{n+1}\right) \\
= & \mathcal{L}_{k}\left(h_{n+1}, u, \ldots, u, h_{1}, \ldots, h_{n}\right) \\
& +\mathcal{L}_{k}\left(u, h_{n+1}, u, \ldots, u, h_{1}, \ldots, h_{n}\right) \\
& +\cdots \\
& +\mathcal{L}_{k}\left(u, \ldots, u, h_{n+1}, h_{1}, \ldots, h_{n}\right)
\end{aligned}
$$

and similarly with all the other terms in (9). We can then compute $D D^{n} \mathcal{M}_{k}=$ $D^{n+1} \mathcal{L}_{k}$, from which the representation (9) follows for $n+1$. Directly from this representation one observes that $D^{n+1} \mathcal{M}_{k}$ can be seen as a map from $B$ to $L_{n+1}(B)$. Finally, $D^{k} \mathcal{M}_{k}(u)$ is constant and therefore $D^{k+1} \mathcal{M}_{k}(u)=0$.

In view of this result, it is fair to call the elements in $M_{k}(B)$ monomials, as we have done.

Corollary 10. Assume for $k \in \mathbb{N}$ that $\mathcal{M}_{k} \in M_{k}(B)$ is induced by $\mathcal{L}_{k} \in L_{k}(B)$. Then the $n$-th Fréchet derivative $D^{n} \mathcal{M}_{k}: B \rightarrow L_{n}(B)$ is symmetric for any $2 \leq$ $n \leq k$, i.e.

$$
D^{n} \mathcal{M}_{k}(u)\left(h_{1}, \ldots, h_{n}\right)=D^{n} \mathcal{M}_{k}(u)\left(h_{\sigma(1)}, \ldots, h_{\sigma(n)}\right)
$$

for any permutation $\sigma \in S_{n}$, where $S_{n}$ denotes the set of permutations on $\{1, \ldots, n\}$.

Proof. We can rewrite (9) as a double sum where we first fix the appearance of the $u$ and then sum over those terms with $u$ in the same coordinate. Again for notational simplicity we look at the specific one with the $u$ fixed to be in the first $k-n$ coordinates and we find that

$$
\begin{aligned}
& \sum_{\substack{x_{i} \in\left\{h_{1}, \ldots, h_{n}\right\} \\
\#\left\{i \mid x_{i}=h_{j}\right\}=1 \\
1 \leq j \leq n}} \mathcal{L}_{k}\left(u, \ldots, u, x_{1}, \ldots, x_{n}\right) \\
& \quad=\sum_{\sigma \in S_{n}} \mathcal{L}_{k}\left(u, \ldots, u, h_{\sigma(1)}, \ldots, h_{\sigma(n)}\right)
\end{aligned}
$$

and therefore the expression is symmetric. The same argument works for any fixed positions for the $u$ 's and there are $\left(\begin{array}{l}k \\ n\end{array}\right)$ possible ways to fix them. Therefore 
$D^{n} \mathcal{M}_{k}(u)\left(h_{1}, \ldots, h_{n}\right)$ is the sum of $\left(\begin{array}{l}k \\ n\end{array}\right)$ symmetric functions and is therefore itself symmetric.

We immediately get the following Corollary which will be important later for polynomials in Banach algebras.

Corollary 11. Let $B$ be a Banach algebra and $\mathcal{L} \in L(B)$. Define the $k$-th order monomial $\mathcal{M}_{k}(u):=\mathcal{L}\left(u^{k}\right)$. For $n \leq k$, the $n$-th order Fréchet derivative $D^{n} \mathcal{M}_{k}$ : $B \rightarrow L_{n}(B)$ of $\mathcal{M}_{k}$ is given by

$$
D^{n} \mathcal{M}_{k}(u)\left(h_{1}, \ldots, h_{n}\right)=\mathcal{L}\left(\sum_{\substack{x_{i} \in\left\{u, h_{1}, \ldots, h_{n}\right\} \\ \#\left\{i \mid x_{i}=h_{j}\right\}=1 \\ 1 \leq j \leq n}} x_{1} \cdots x_{k}\right) .
$$

Furthermore if $B$ is commutative, then the expression simplifies to

$$
D^{n} \mathcal{M}_{k}(u)\left(h_{1}, \ldots, h_{n}\right)=\frac{k !}{(k-n) !} \mathcal{L}\left(h_{1} \cdots h_{n} u^{k-n}\right) .
$$

Proof. The monomial $\mathcal{M}_{k}$ is induced from the multilinear map $\mathcal{L}_{k}:\left(u_{1}, \ldots, u_{k}\right) \rightarrow$ $\mathcal{L}\left(u_{1} \cdots u_{k}\right)$. Then $(12)$ directly follows form Proposition 9. If $B$ is commutative, then all terms appearing in the sum in $(12)$ are equal. In fact there are $\left(\begin{array}{l}k \\ n\end{array}\right)$ ways to fix the appearance of the $u$ and then $n$ ! ways to distribute the $h_{1}, \ldots, h_{n}$ in the remaining positions. So altogether there are $\frac{k !}{(k-n) !}$ equal terms and $(13)$ follows.

2.1. Multilinear forms. In this section we shall elaborate a bit on multilinear forms which map into the field $\mathbb{F}$ instead of the Banach space $B$. We first give a precise definition:

Definition 12. We say that $\mathcal{L}_{k}: B^{k} \rightarrow \mathbb{F}$ for $k \in \mathbb{N}$ is a $k$-linear form if it is linear in each argument in the sense that for any $x_{1}, x_{2}, \ldots, x_{k}, y \in B$ and $a, b \in \mathbb{F}$

$$
\begin{aligned}
\mathcal{L}_{k}\left(x_{1}, \ldots, x_{j-1}, a x_{j}+b y, x_{j+1}, \ldots, x_{k}\right) \\
\quad=a \mathcal{L}_{k}\left(x_{1}, \ldots, x_{k}\right)+b \mathcal{L}_{k}\left(x_{1}, \ldots, x_{j-1}, y, x_{j+1}, \ldots, x_{k}\right)
\end{aligned}
$$

for each $j=1, \ldots, k$. A k-linear form $\mathcal{L}_{k}$ is bounded if there exists a constant $K>0$ such that

$$
\left|\mathcal{L}_{k}\left(x_{1}, \ldots, x_{k}\right)\right| \leq K\left\|x_{1}\right\| \cdots\left\|x_{k}\right\|
$$

for all $x_{1}, \ldots, x_{k} \in B$. We denote the space of bounded $k$-linear forms by $L_{k}^{\mathbb{F}}(B)$.

Notice that $L_{1}^{\mathbb{F}}(B)$ is the dual space of $B$. A $k$-linear form $\mathcal{L}_{k}$ induces a $k$ monomial $\mathcal{M}_{k}: B \rightarrow \mathbb{F}$ by

$$
\mathcal{M}_{k}(x):=\mathcal{L}_{k}(x, \ldots, x) .
$$

If $\mathcal{L}_{k} \in L_{k}^{\mathbb{F}}(B)$, we see that $\left|\mathcal{M}_{k}(x)\right| \leq K\|x\|^{k}$, and we denote the set of all such $k$-monomials by $M_{k}^{\mathbb{F}}(B)$. We use the convention that $M_{0}^{\mathbb{F}}(B)=\mathbb{F}$. Observe that $M_{1}^{\mathbb{F}}(B)=L_{1}^{\mathbb{F}}(B)$.

We show that the multilinearity preserving property implies that also monomials arising from multilinear forms are preserved. We use this result in Section 4 for the calculation of conditional moments for Hilbert space valued stochastic processes but the result might be of independent interest. 
Proposition 13. Let $B$ be a Hilbert space with inner product $\langle\cdot, \cdot\rangle$. Let $(X(t))_{t \geq 0}$ be a multilinear $B$-valued process with respect to the family of $B$-valued random variables $(X(s ; t))_{0 \leq s \leq t<\infty}$. For every $k$-monomial $\mathcal{M}_{k} \in M_{k}^{\mathbb{F}}(B)$, there exist $j$ monomials $\overline{\mathcal{M}}_{j} \in M_{j}^{\mathbb{F}}(B), j=0, \ldots, k$ such that

$$
\mathbb{E}\left[\mathcal{M}_{k}(X(t)) \mid \mathcal{F}_{s}\right]=\sum_{j=0}^{k} \overline{\mathcal{M}}_{j}(X(s ; t)) .
$$

Proof. Let $\mathcal{M}_{k} \in M_{k}^{\mathbb{F}}(B)$. Choose $z \in B$ with $\|z\|_{B}=1$. Define the $k$-monomial $\mathcal{M}_{k}^{z} \in M_{k}(B)$ by $\mathcal{M}_{k}^{z}(x):=z \mathcal{M}_{k}(x)$. Then there exist $\overline{\mathcal{M}}_{j}^{z} \in M_{j}(B)$ for $j=$ $0, \ldots, k$ such that

$$
\mathbb{E}\left[\mathcal{M}_{k}^{z}(X(t)) \mid \mathcal{F}_{s}\right]=\sum_{j=0}^{k} \overline{\mathcal{M}}_{j}^{z}(X(s ; t)) .
$$

Clearly, $\overline{\mathcal{M}}_{j}:=\left\langle\overline{\mathcal{M}}_{j}^{z}(x), z\right\rangle$ defines an element in $M_{j}^{\mathbb{F}}(B)$. Now observe that

$$
\begin{aligned}
\mathbb{E}\left[\mathcal{M}_{k}(X(t)) \mid \mathcal{F}_{s}\right] & =\left\langle\mathbb{E}\left[\mathcal{M}_{k}^{z}(X(t)) \mid \mathcal{F}_{s}\right], z\right\rangle \\
& =\left\langle\sum_{j=0}^{k} \overline{\mathcal{M}}_{j}^{z}(X(s ; t)), z\right\rangle \\
& =\sum_{j=0}^{k} \overline{\mathcal{M}}_{j}(X(s ; t)) .
\end{aligned}
$$

\section{Multiplichtive Maps AND POLYNOMials}

We shall now focus on Banach spaces $B$ that are also Banach algebras. We recall that when $B$ is a Banach algebra, there is a multiplication operator $\cdot: B \times B \rightarrow B$ defined such that $(B,+, \cdot)$ is an associative $\mathbb{F}$-algebra and $\|x \cdot y\| \leq\|x\| \cdot\|y\|$ for any $x, y \in B$.

Suppose that $(X(t))_{t \geq 0}$ is an independent increment process in $B$ (see Definition [6), and recall the decomposition $X(t)=X^{\perp}(s ; t)+X^{\|}(s ; t)$ for any $0 \leq$ $s \leq t$. According to Theorem 8, $(X(t))_{t>0}$ is a multilinear process with respect to $\left(X^{\|}(s ; t)\right)_{0 \leq s \leq t<\infty}$, i.e., for every $\mathcal{M}_{k} \in M_{k}(B), k \in \mathbb{N}$, there exist $\overline{\mathcal{M}}_{j} \in$ $M_{j}(B), j=0, \ldots, k$ such that

$$
\mathbb{E}\left[\mathcal{M}_{k}(X(t)) \mid \mathcal{F}_{s}\right]=\sum_{j=0}^{k} \overline{\mathcal{M}}_{j}\left(X^{\|}(s ; t)\right),
$$

for all $0 \leq s \leq t$. We now look at the particular case of $\mathcal{M}_{k}(x)=x^{k}$ and address the question under which conditions the induced $j$-order monomials $\overline{\mathcal{M}}_{j}$ in 15 are of polynomial type as well.

Lemma 14. Assume that $B$ is a commutative Banach algebra. Then for all $0 \leq$ $s \leq t$

with

$$
\mathbb{E}\left[X^{k}(t) \mid \mathcal{F}_{s}\right]=q_{k}\left(X^{\|}(s ; t)\right)
$$

$$
q_{k}(x)=\sum_{n=0}^{k}\left(\begin{array}{l}
k \\
n
\end{array}\right) \mathbb{E}\left[\left(X^{\perp}(s ; t)\right)^{k-n}\right] x^{n} .
$$


Here, we use the convention that $x^{0}=1 \in \mathbb{F}$, that is, the term $b_{0} x^{0}=b_{0} \in B$.

Proof. Let $k \in \mathbb{N}$. By the binomial formula, we find for $s \leq t$

$$
X^{k}(t)=\left(X^{\perp}(s ; t)+X^{\|}(s ; t)\right)^{k}=\sum_{n=0}^{k}\left(\begin{array}{l}
k \\
n
\end{array}\right)\left(X^{\perp}(s ; t)\right)^{k-n}\left(X^{\|}(s ; t)\right)^{n} .
$$

We get by $\mathcal{F}_{t^{-}}$-adaptedness of the process and Lemma 25 (Appendix A that $\left(X^{\|}(s ; t)\right)^{n}$ is strongly $\mathcal{F}_{s}$-measurable for all $n \leq k$. From Proposition 26 (Appendix A),

$$
\begin{aligned}
\mathbb{E}\left[X^{k}(t) \mid \mathcal{F}_{s}\right] & =\sum_{n=0}^{k}\left(\begin{array}{l}
k \\
n
\end{array}\right) \mathbb{E}\left[\left(X^{\perp}(s ; t)\right)^{k-n}\left(X^{\|}(s ; t)\right)^{n} \mid \mathcal{F}_{s}\right] \\
& =\sum_{n=0}^{k}\left(\begin{array}{l}
k \\
n
\end{array}\right) \mathbb{E}\left[\left(X^{\perp}(s ; t)\right)^{k-n} \mid \mathcal{F}_{s}\right]\left(X^{\|}(s ; t)\right)^{n} \\
& =\sum_{n=0}^{k}\left(\begin{array}{l}
k \\
n
\end{array}\right) \mathbb{E}\left[\left(X^{\perp}(s ; t)\right)^{k-n}\right]\left(X^{\|}(s ; t)\right)^{n} .
\end{aligned}
$$

In the last step, we used that $X^{\perp}(s ; t)$ is independent of $\mathcal{F}_{s}$ and in the first step Lemma 22 (Appendix A). Thus, the lemma follows.

If $B$ is commutative, define a polynomial $p_{k}: B \rightarrow B$ of order $k \in \mathbb{N}$, as

$$
p_{k}(x)=\sum_{n=0}^{k} b_{n} x^{n},
$$

where $\left(b_{n}\right)_{n=0}^{k} \subset B$ and with the convention that $x^{0}=1 \in \mathbb{F}$, i.e., $b_{0} x^{0}=b_{0} \in B$. If $x \in B$, we find

$$
\left\|p_{k}(x)\right\| \leq \sum_{n=0}^{k}\left\|b_{n}\right\|\|x\|^{n}<\infty
$$

by the triangle inequality and Banach algebra norm. We denote the space of polynomials in $B$ of order $k$ by $\mathrm{Pol}_{k}(B)$. If $B=\mathbb{R}, \mathrm{Pol}_{k}(\mathbb{R})$ is the space of polynomials on the real line of order $k$.

From Lemma 14 it is simple to see that in a commutative Banach algebra

$$
\mathbb{E}\left[p_{k}(X(t)) \mid \mathcal{F}_{s}\right]=\sum_{n=0}^{k} b_{n} q_{n}\left(X^{\|}(s ; t)\right)=\widetilde{q}_{k}\left(X^{\|}(s ; t)\right)
$$

for any $k \in \mathbb{N}$ and $0 \leq s \leq t$, where $\widetilde{q}_{k} \in \operatorname{Pol}_{k}(B)$ is given by

$$
\widetilde{q}_{k}(x)=\sum_{n=0}^{k} b_{n} \sum_{j=0}^{n}\left(\begin{array}{l}
n \\
j
\end{array}\right) \mathbb{E}\left[\left(X^{\perp}(s ; t)\right)^{n-j}\right] x^{j} .
$$

This motivates a definition of a polynomial process in a commutative Banach algebra $B$ :

Definition 15. Let $(X(t))_{t \geq 0}$ be a B-valued stochastic process where $B$ is a $B a$ nach algebra. Furthermore, let $(X(s ; t))_{0 \leq s \leq t<\infty}$ be a family of $B$-valued random variables, such that $X(s ; t)$ is strongly $\mathcal{F}_{s}$-measurable for all $0 \leq s \leq t$. The 
process $(X(t))_{t \geq 0}$ is said to be a polynomial process with respect to the family $(X(s ; t))_{0 \leq s \leq t<\infty}$ if for all $s \leq t, k \in \mathbb{N}$ and every polynomial $p_{k} \in \operatorname{Pol}_{k}(B)$ there exists a polynomial $q_{m} \in \operatorname{Pol}_{m}(B), m \leq k$ such that

$$
\mathbb{E}\left[p_{k}(X(t)) \mid \mathcal{F}_{s}\right]=q_{m}(X(s ; t)) .
$$

Note that the coefficients of $q_{m}$ may depend on the times $s$ and $t$. We summarize our findings from above in the following Theorem.

Theorem 16. Assume that $B$ is a commutative Banach algebra. Then the independent increment process $(X(t))_{t \geq 0}$ defined in Definition 6 is a polynomial process with respect to $\left(X^{\|}(s ; t)\right)_{0 \leq s \leq t<\infty}$.

Observe that any $b \in B$ gives rise to a multiplication operator $B \ni x \mapsto b x \in B$, being a bounded linear operator. Hence, the above definition of polynomials may be viewed as a special case of more general polynomials with $b_{n} \in L(B)$, as we define next. Define a generalized polynomial $\mathcal{P}_{k}: B \rightarrow B$ of order $k \in \mathbb{N}$, as

$$
\mathcal{P}_{k}(x)=\sum_{n=0}^{k} \mathcal{B}_{n}\left(x^{n}\right),
$$

where $\left(\mathcal{B}_{n}\right)_{n=1}^{k} \subset L(B)$ and $\mathcal{B}_{0} \in B$ is a constant reflecting the fact that $x^{0}=1 \in \mathbb{F}$. If $x \in B$, we find

$$
\left\|\mathcal{P}_{k}(x)\right\| \leq \sum_{n=0}^{k}\left\|\mathcal{B}_{n}\right\|_{\text {op }}\|x\|^{n}<\infty
$$

by the triangle inequality and Banach algebra norm. Here, $\left\|\mathcal{B}_{n}\right\|_{\text {op }}$ denotes the operator norm of $\mathcal{B}_{n}$. We denote the space of generalized polynomials in $B$ of order $k$ by $\mathrm{gPol}_{k}(B)$. If $B=\mathbb{R}, \mathrm{gPol}_{k}(\mathbb{R})=\mathrm{Pol}_{k}(\mathbb{R})$ is the space of polynomials on the real line of order $k$.

We define the following

Definition 17. Let $(X(t))_{t \geq 0}$ be a B-valued stochastic process where $B$ is a Banach algebra. Furthermore, let $(X(s ; t))_{0 \leq s \leq t<\infty}$ be a family of $B$-valued random variables, such that $X(s ; t)$ is strongly $\mathcal{F}_{s}$-measurable for all $0 \leq s \leq t$. The process $(X(t))_{t>0}$ is said to be a generalized polynomial process with respect to the family $(X(s ; t))_{0 \leq s \leq t<\infty}$ if for all $s \leq t, k \in \mathbb{N}$ and every generalized polynomial $\mathcal{P}_{k} \in g_{\text {Pol }}(B)$ there exists a generalized polynomial $\mathcal{Q}_{m} \in g P o l_{m}(B), m \leq k$ such that

$$
\mathbb{E}\left[\mathcal{P}_{k}(X(t)) \mid \mathcal{F}_{s}\right]=\mathcal{Q}_{m}(X(s ; t)) .
$$

Note that in Definition 15 and 17 we do not assume that $B$ is commutative. The name generalized polynomial process is justified by the following Proposition which states that every polynomial process is also a generalized polynomial process.

Proposition 18. Assume $B$ is a Banach algebra, and $(X(t))_{t \geq 0}$ is a polynomial process in $B$ with respect to the family $(X(s ; t))_{0 \leq s \leq t<\infty}$. Then $(X(t))_{t \geq 0}$ is also a generalized polynomial process in $B$ with respect to the family $(X(s ; t))_{0 \leq s \leq t<\infty}$.

Proof. For $k \in \mathbb{N}$, let $\mathcal{P}_{k} \in \mathrm{gPol}_{m}(B)$ with

$$
\mathcal{P}_{k}(x)=\sum_{n=0}^{k} \mathcal{B}_{n}\left(x^{n}\right) .
$$


Because $(X(t))_{t \geq 0}$ is a polynomial process with respect to $(X(s ; t))_{0 \leq s \leq t<\infty}$, it follows that for each $0 \leq n \leq k$ there exists a $q_{m(n)} \in \operatorname{Pol}_{m(n)}(B)$ for $m(n) \leq k$ such that for any $s \leq t$

$$
\mathbb{E}\left[(X(t))^{n} \mid \mathcal{F}_{s}\right]=q_{m(n)}(X(s ; t))=\sum_{j=0}^{m(n)} b_{n, j}(X(s ; t))^{j}
$$

where $\left(b_{n, j}\right)_{j=0, \ldots, m(n)} \subset B$. It follows using Lemma 20 that

$$
\begin{aligned}
\mathbb{E}\left[\mathcal{B}_{n}\left((X(t))^{n}\right) \mid \mathcal{F}_{s}\right] & =\mathcal{B}_{n}\left(\mathbb{E}\left[\left((X(t))^{n}\right) \mid \mathcal{F}_{s}\right]\right) \\
& =\mathcal{B}_{n}\left(\sum_{j=0}^{m(n)} b_{n, j}(X(s ; t))^{j}\right) \\
& =\sum_{j=0}^{m(n)} \mathcal{B}_{n}\left(b_{n, j}(X(s ; t))^{j}\right) \\
& =\sum_{j=0}^{m(n)} \widetilde{\mathcal{B}}_{n, j}(X(s ; t))^{j}
\end{aligned}
$$

with $\widetilde{\mathcal{B}}_{n, j} \in L(B)$ being defined by $B \ni x \mapsto \mathcal{B}_{n}\left(b_{n, j} x\right) \in B$. Define the generalized polynomial $\mathcal{Q}_{\widehat{m}} \in \operatorname{gPol}_{\widehat{m}}(B)$ by $\mathcal{Q}_{\widehat{m}}(x)=\sum_{n=0}^{k} \sum_{j=0}^{m(n)} \widetilde{\mathcal{B}}_{n, j} x^{j}$ with $\widehat{m}:=$ $\max _{0 \leq n \leq k} m(n) \leq k$. The result follows.

To see that the opposite does not hold in general we return to Example 7 and now assume that $B$ is a separable Hilbert space with a commutative algebra defined.

As before we decompose $X(t)$ as defined in (4) into $X^{\perp}(s ; t):=\int_{s}^{t} \mathcal{S}_{t-u} d W(u)$ and $X^{\|}(s ; t):=\int_{0}^{s} \mathcal{S}_{t-u} d W(u)$ where $X^{\perp}(s ; t)$ is independent of $\mathcal{F}_{s}$ and $X^{\|}(s ; t)$ is $\mathcal{F}_{s}$-measurable. Hence, $(X(t))_{t \geq 0}$ is an independent increment process in $B$ and by Theorem 16, it holds for any $k \in \mathbb{N}$ and $s \leq t$

$$
\mathbb{E}\left[X^{k}(t) \mid \mathcal{F}_{s}\right]=\sum_{n=0}^{k}\left(\begin{array}{l}
k \\
n
\end{array}\right) \mathbb{E}\left[\left(X^{\perp}(s ; t)\right)^{k-n}\right]\left(X^{\|}(s ; t)\right)^{n} .
$$

Hence, as expected, $(X(t))_{t \geq 0}$ is a polynomial process with respect to the family $\left(X^{\|}(s ; t)\right)_{0 \leq s \leq t<\infty}$. Let us analyse the situation a few steps further: From the semigroup property of $\left(\mathcal{S}_{t}\right)_{t \geq 0}$, we find that

$$
X^{\|}(s ; t)=\mathcal{S}_{t-s} \int_{0}^{s} \mathcal{S}_{s-u} d W(u)=\mathcal{S}_{t-s} X(s) .
$$

Thus,

$$
\mathbb{E}\left[X^{k}(t) \mid \mathcal{F}_{s}\right]=\sum_{n=0}^{k}\left(\begin{array}{l}
k \\
n
\end{array}\right) \mathbb{E}\left[\left(X^{\perp}(s ; t)\right)^{k-n}\right]\left(\mathcal{S}_{t-s} X(s)\right)^{n} .
$$

Now, assume $\left(\mathcal{S}_{t}\right)_{t \geq 0}$ is an algebra homomorphism so that $\mathcal{S}_{t}(x \cdot y)=\left(\mathcal{S}_{t} x\right) \cdot\left(\mathcal{S}_{t} y\right)$ for any $x, y \in B$. Then $\left(\mathcal{S}_{t} x\right)^{n}=\mathcal{S}_{t} x^{n}$ for all $n \in \mathbb{N}$ and we find

$$
\mathbb{E}\left[X^{k}(t) \mid \mathcal{F}_{s}\right]=\sum_{n=0}^{k}\left(\begin{array}{l}
k \\
n
\end{array}\right) \mathbb{E}\left[\left(X^{\perp}(s ; t)\right)^{k-n}\right] \mathcal{S}_{t-s} X^{n}(s) .
$$


This shows that $(X(t))_{t \geq 0}$ is a generalized polynomial process with respect to the family $(X(s))_{0 \leq s \leq t<\infty}$. This is in line with the definition of finite-dimensional polynomial processes (see Cuchiero et al. 9 and Filipović and Larsson [13]), and the fact that we can establish the generalized polynomial preserving property of the process with respect to itself is significantly stronger and more applicable than merely in terms of some family of $\mathcal{F}_{s}$-measurable random variables. On the other hand, $(X(t))_{t \geq 0}$ is in general not a polynomial process with respect to $(X(s))_{0 \leq s \leq t<\infty}$, as the coefficients on the right hand side of $(20)$ are elements in $L(B)$. In fact, when $B$ is a function space with multiplication defined point-wise and $\mathcal{S}_{t}$ is the shift operator (such an example is considered in more detail in Section 4.2), then for instance $\mathbb{E}\left[X(t) \mid \mathcal{F}_{s}\right]=\mathcal{S}_{t-s} X(s)=X(s)(t-s+\cdot)$. However, if $(X(t))_{t>0}$ is polynomial, then for every $y:=t-s$ there must exist $a_{y}, b_{y} \in B$ such that $\mathbb{E}\left[X(t) \mid \mathcal{F}_{s}\right]=X(s)(t-s+\cdot)=X(s)(y+\cdot)=a_{y} X(s)(\cdot)+b_{y}$. Since $\mathcal{S}_{t-s}$ is linear we may conclude that $b_{y}=0$. Then evaluating at 0 leads in particular to $X(s)(y)=a_{y}(0) X(s)(0)$ This implies that $X(s)(\cdot)$ is measurable with respect to the sigma algebra $\sigma(X(s)(0))$. However, this cannot be the case if the driving Wiener process $(W(t))_{t \geq 0}$ is varying with $x$. This provides us with an example of a process that is generalized polynomial but not polynomial.

It is worth emphasising that the above analysis shows that, in general, stochastic convolutions as in (4) are polynomial processes with respect to $\left(\mathcal{S}_{t-s} X(s)\right)_{0 \leq s \leq t<\infty}$. Indeed, they are generalized polynomial processes with respect to $(X(s))_{0 \leq s \leq t<\infty}$ when the semigroup is a homomorphism, but not necessarily polynomial with respect to the same family. Hence, the extension of Ornstein-Uhlenbeck processes to infinite dimensions as in (5) is not straightforwardly preserving the natural polynomial property from the finite-dimensional case.

The class of processes defined in (4) is of interest from the application point of view. Stochastic evolution equations like the Ornstein-Uhlenbeck process in (5) appear in many applications, for example as the heat equation in random media (see e.g. Walsh 20 ) or as the dynamics of forward prices in finance and commodity markets (see e.g. Benth and Krühner [4). We return to the latter in the next Section.

3.1. Counterexample: non-commutative case. Consider the case when $B$ is a non-commutative Banach algebra. Then the binomial formula used in the proof of Lemma 14 and later above does not hold. For example, if $(X(t))_{t \geq 0}$ is an independent increment process in $B$, we find for $t \geq s$ that

$$
\begin{aligned}
\mathbb{E}\left[X^{3}(t) \mid \mathcal{F}_{s}\right]= & \mathbb{E}\left[\left(X^{\perp}(s ; t)\right)^{3} \mid \mathcal{F}_{s}\right]+\mathbb{E}\left[\left(X^{\perp}(s ; t)\right)^{2} X^{\|}(s ; t) \mid \mathcal{F}_{s}\right] \\
& +\mathbb{E}\left[\left(X^{\perp}(s ; t)\right) X^{\|}(s ; t)\left(X^{\perp}(s ; t)\right) \mid \mathcal{F}_{s}\right]+\mathbb{E}\left[\left(X^{\perp}(s ; t)\right)\left(X^{\|}(s ; t)\right)^{2} \mid \mathcal{F}_{s}\right] \\
& +\mathbb{E}\left[X^{\|}(s ; t)\left(X^{\perp}(s ; t)\right)^{2} \mid \mathcal{F}_{s}\right]+\mathbb{E}\left[X^{\|}(s ; t)\left(X^{\perp}(s ; t)\right) X^{\|}(s ; t) \mid \mathcal{F}_{s}\right] \\
& +\mathbb{E}\left[\left(X^{\|}(s ; t)\right)^{2}\left(X^{\perp}(s ; t)\right) \mid \mathcal{F}_{s}\right]+\mathbb{E}\left[\left(X^{\|}(s ; t)\right)^{3} \mid \mathcal{F}_{s}\right] \\
= & \mathbb{E}\left[\left(X^{\perp}(s ; t)\right)^{3}\right]+E\left[\left(X^{\perp}(s ; t)\right)^{2}\right] X^{\|}(s ; t) \\
& +\mathbb{E}\left[\left(X^{\perp}(s ; t)\right) X^{\|}(s ; t)\left(X^{\perp}(s ; t)\right) \mid \mathcal{F}_{s}\right] \\
& +\mathbb{E}\left[\left(X^{\perp}(s ; t)\right)\right]\left(X^{\|}(s ; t)\right)^{2}+X^{\|}(s ; t) \mathbb{E}\left[\left(X^{\perp}(s ; t)\right)^{2}\right] \\
& +\left(X^{\|}(s ; t)\right)^{2} \mathbb{E}\left[\left(X^{\perp}(s ; t)\right)\right]+\left(X^{\|}(s ; t)\right)^{3}
\end{aligned}
$$


after appealing to independence and measurability using Lemmas 22 and 26 (Appendix A. It is not immediately clear how to deal with the term involving the conditional expectation of $X^{\perp}(s ; t) X^{\|}(s ; t) X^{\perp}(s ; t)$, and thus how to express $\mathbb{E}\left[X^{3}(t) \mid \mathcal{F}_{s}\right]$ as a polynomial in $X^{\|}(s ; t)$.

Using Proposition 23 (Appendix A), we know that

$$
\mathbb{E}\left[\left(X^{\perp}(s ; t)\right) X^{\|}(s ; t)\left(X^{\perp}(s ; t)\right) \mid \mathcal{F}_{s}\right]=\mathbb{E}\left[\left(X^{\perp}(s ; t)\right) y\left(X^{\perp}(s ; t)\right)\right]_{y=X \|}(s ; t)
$$

and observe that $B \ni y \mapsto \mathbb{E}\left[\left(X^{\perp}(s ; t)\right) y\left(X^{\perp}(s ; t)\right)\right] \in B$ is a linear function. Furthermore, it is bounded as

$$
\begin{aligned}
\left\|\mathbb{E}\left[\left(X^{\perp}(s ; t)\right) y\left(X^{\perp}(s ; t)\right)\right]\right\| & \leq \mathbb{E}\left[\left\|\left(X^{\perp}(s ; t)\right) y\left(X^{\perp}(s ; t)\right)\right\|\right] \\
& \leq \mathbb{E}\left[\left\|\left(X^{\perp}(s ; t)\right)\right\|\|y\|\left\|\left(X^{\perp}(s ; t)\right)\right\|\right] \\
& =\|y\| \mathbb{E}\left[\left\|\left(X^{\perp}(s ; t)\right)\right\|^{2}\right] .
\end{aligned}
$$

Altogether this means that

$$
\mathbb{E}\left[X^{\perp}(s ; t) X^{\|}(s ; t) X^{\perp}(s ; t) \mid \mathcal{F}_{s}\right]=\mathcal{L}\left(X^{\|}(s ; t)\right)
$$

for the bounded operator $\mathcal{L} \in L(B)$ defined by $y \mapsto \mathbb{E}\left[X^{\perp}(s ; t) y X^{\perp}(s ; t)\right]$. To show that in fact $\mathcal{L}$ is not a (left)-multiplication operator we look at the vector space $\mathbb{R}^{2 \times 2}$ of $2 \times 2$-matrices equipped with a sub-multiplicative matrix norm and the usual matrix product. This space is well-known to be a non-commutative Banach algebra. Let now $\left(L_{i j}(t)\right)_{t \geq 0}$ for $i, j=1,2$ be 4 independent copies of the real-valued Lévy processes $(L(t))_{t \geq 0}$ with finite moments of all orders. Then

$$
X(t)=\left(\begin{array}{ll}
L_{11}(t) & L_{12}(t) \\
L_{21}(t) & L_{22}(t)
\end{array}\right)
$$

defines an independent increment process in the space of $2 \times 2$-matrices. It follows that

$$
X^{\perp}(s ; t)=\left(\begin{array}{cc}
\Delta_{s, t} L_{11} & \Delta_{s, t} L_{12} \\
\Delta_{s, t} L_{21} & \Delta_{s, t} L_{22}
\end{array}\right)
$$

where $\Delta_{s, t} L_{i j}(t)=L_{i j}(t)-L_{i j}(s)$ for $s \leq t$. Choose now

$$
h:=\left(\begin{array}{ll}
0 & 1 \\
0 & 0
\end{array}\right)
$$

We obtain that

$$
\mathcal{L}(h)=\left(\begin{array}{cc}
\mathbb{E}\left[\Delta_{s, t} L\right]^{2} & \mathbb{E}\left[\Delta_{s, t} L\right]^{2} \\
\mathbb{E}\left[\left(\Delta_{s, t} L\right)^{2}\right] & \mathbb{E}\left[\Delta_{s, t} L\right]^{2}
\end{array}\right)=\left(\begin{array}{ll}
0 & 0 \\
1 & 0
\end{array}\right)=: g
$$

if $L$ is such that $\mathbb{E}[L(t)]=0$ and $s<t$ such that $\mathbb{E}\left[\left(\Delta_{s, t} L\right)^{2}\right]=1$, where $\Delta_{s, t} L=$ $L(t)-L(s)$. However, one easily verifies that no matrix $a \in \mathbb{R}^{2 \times 2}$ exists with $a h=g$.

This shows that the independent increment process $(X(t))_{t \geq 0}$ is not a polynomial process in a non-commutative Banach algebra $B$. This is very different from the commutative case, where we recall from Theorem 16 that independent increment processes are polynomial processes.

Motivated by the above derivation, we may ask the question whether $(X(t))_{t \geq 0}$ is a generalized polynomial process. However, this is also not the case as can be 
seen by looking at $\mathbb{E}\left[X^{5}(t) \mid \mathcal{F}_{s}\right]$ : Similar calculation as above yields one term of the form

$$
\begin{aligned}
& \mathbb{E}\left[X^{\perp}(s ; t) X^{\|}(s ; t) X^{\perp}(s ; t) X^{\|}(s ; t) X^{\perp}(s ; t) \mid \mathcal{F}_{s}\right] \\
& =\mathbb{E}\left[X^{\perp}(s ; t) y X^{\perp}(s ; t) y X^{\perp}(s ; t)\right]_{y=X \|}(s ; t)
\end{aligned}
$$

and the question is whether this expression can be written as $\mathcal{L}_{2}\left(\left(X^{\|}(s ; t)\right)^{2}\right)+$ $\mathcal{L}_{1}\left(\left(X^{\|}(s ; t)\right)\right)+b$ for some $\mathcal{L}_{1}, \mathcal{L}_{2} \in L(B), b \in B$. Let us assume that this is indeed the case, that is,

$$
f(y):=\mathbb{E}\left[X^{\perp}(s ; t) y X^{\perp}(s ; t) y X^{\perp}(s ; t)\right]=\mathcal{L}_{2}\left(y^{2}\right)+\mathcal{L}_{1}(y)+b .
$$

By Proposition 9 we know that

$$
\begin{aligned}
D^{2} f(y)\left(h_{1}, h_{2}\right)=\mathbb{E}\left[X^{\perp}(s ; t) h_{1} X^{\perp}(s ; t) h_{2} X^{\perp}(s ; t)\right] \\
+\mathbb{E}\left[X^{\perp}(s ; t) h_{2} X^{\perp}(s ; t) h_{1} X^{\perp}(s ; t)\right]
\end{aligned}
$$

and by Corollary 11 that

$$
D^{2}\left(\mathcal{L}_{2}\left(y^{2}\right)\right)\left(h_{1}, h_{2}\right)=\mathcal{L}_{2}\left(h_{1} h_{2}\right)+\mathcal{L}_{2}\left(h_{2} h_{1}\right) .
$$

and $D^{2}\left(\mathcal{L}_{1}+b\right)=0$. If $f(y)=\mathcal{L}_{2}\left(y^{2}\right)+\mathcal{L}_{1}(y)+b$ then of course also their derivatives agree and

$$
D^{2} f(y)\left(h_{1}, h_{2}\right)=D^{2}\left(\mathcal{L}_{2}\left(y^{2}\right)\right)\left(h_{1}, h_{2}\right)
$$

for every $h_{1}, h_{2} \in B$.

We now choose

$$
h_{1}:=\left(\begin{array}{cc}
1 & 0 \\
0 & 0
\end{array}\right) \quad h_{2}:=\left(\begin{array}{cc}
0 & 0 \\
0 & 1
\end{array}\right)
$$

and first observe that $h_{1} \cdot h_{2}=h_{2} \cdot h_{1}=\mathbf{0}_{2}$ with $\mathbf{0}_{2}$ being the $2 \times 2$-matrix of zeros, then $D^{2}\left(\mathcal{L}_{2}\left(y^{2}\right)\right)\left(h_{1}, h_{2}\right)=\mathcal{L}_{2}\left(\mathbf{0}_{2}\right)+\mathcal{L}_{2}\left(\mathbf{0}_{2}\right)=\mathbf{0}_{2}$ independent of the specification of $(X(t))_{t \geq 0}$.

With the same choice for $(X(t))_{t \geq 0}$ as above we derive,

$$
\begin{aligned}
& D^{2} f(y)\left(h_{1}, h_{2}\right) \\
= & \left(\begin{array}{cc}
2 \mathbb{E}\left[\Delta_{s, t} L\right]^{3} & \mathbb{E}\left[\Delta_{s, t} L\right]^{3}+\mathbb{E}\left[\left(\Delta_{s, t} L\right)^{2}\right] \mathbb{E}\left[\Delta_{s, t} L\right] \\
\mathbb{E}\left[\Delta_{s, t} L\right]^{3}+\mathbb{E}\left[\left(\Delta_{s, t} L\right)^{2}\right] \mathbb{E}\left[\Delta_{s, t} L\right] & 2 \mathbb{E}\left[\Delta_{s, t} L\right]^{3}
\end{array}\right) \neq \mathbf{0}_{2}
\end{aligned}
$$

whenever $\mathbb{E}[L(t)] \neq 0$. Choosing now a real-valued Lévy process with mean unequal to zero yields a contradiction to $D^{2}\left(\mathcal{L}_{2}\left(y^{2}\right)\right)\left(\bar{h}_{1}, \bar{h}_{2}\right)=\mathbf{0}_{2}$. So, in general independent increment processes fail to be even generalized polynomial processes in a non-commutative Banach algebra. This shows that even for general Banach algebras one must introduce monomials in $M_{k}(B)$ as the structure preserving class to extend the notion of "polynomial processes" to infinite dimensions, and not merely polynomials nor generalized polynomials.

\section{Applications}

In this section we want to elaborate a bit more on some of the possible applications. 
4.1. Calculation of moments. For multilinear processes in Hilbert space we can compute conditional moments of the norm of the process. To this end, suppose $B$ is a Hilbert space with inner product $\langle\cdot, \cdot\rangle$. Define for $k \in \mathbb{N}$,

$$
\mathcal{L}_{2 k}\left(x_{1}, y_{1}, \ldots, x_{k}, y_{k}\right):=\left\langle x_{1}, y_{1}\right\rangle \cdots\left\langle x_{k}, y_{k}\right\rangle .
$$

$\mathcal{L}_{2 k}$ is a multilinear form, which is obviously bounded. We have

$$
\mathcal{M}_{2 k}(x):=\|x\|^{2 k}
$$

for any $x \in B$. Thus, if $X$ is a multilinear process with respect to the family of $B$-valued random variables $(X(s ; t))_{0 \leq s \leq t<\infty}$, then by Proposition 13 .

$$
\left.\left.\mathbb{E}\left[\|X(t)\|^{2 k} \mid \mathcal{F}_{s}\right]\right]=\mathbb{E}\left[\mathcal{M}_{2 k}(X(t)) \mid \mathcal{F}_{s}\right]\right]=\sum_{j=1}^{2 k} \overline{\mathcal{M}}_{j}(X(s ; t)),
$$

for some $j$ th-order monomials $\overline{\mathcal{M}}_{j}: B \rightarrow \mathbb{K}, j=1, \ldots, 2 k$. So, we can compute even moments of the norm of $X$ in terms of multilinear forms operating on $X(s ; t)$ of order at most $2 k$, where $X(s ; t)$ is $\mathcal{F}_{s}$-measurable.

For the odd moments, we note that for $k=0,1,2, \ldots$, it obviously holds that $2 k+1=\alpha(k) \times(2 k+2)$ for $\alpha(k)=(2 k+1) /(2 k+2) \in(1 / 2,1)$. One has that (see Applebaum [2], page 80)

$$
u^{\alpha(k)}=\frac{\alpha(k)}{\Gamma(1-\alpha(k))} \int_{0}^{\infty}\left(1-e^{-u x}\right) x^{-\alpha(k)-1} d x
$$

Thus, we find the representation

$\mathbb{E}\left[\|X(t)\|^{2 k+1} \mid \mathcal{F}_{s}\right]=\frac{\alpha(k)}{\Gamma(1-\alpha(k))} \int_{0}^{\infty}\left(1-\mathbb{E}\left[\exp \left(-x\|X(t)\|^{2 k+2}\right) \mid \mathcal{F}_{s}\right]\right) x^{-1-\alpha(k)} d x$

Doing a series representation of the exponential function inside the integral on the right hand side, we find that

$\mathbb{E}\left[\|X(t)\|^{2 k+1} \mid \mathcal{F}_{s}\right]=\frac{\alpha(k)}{\Gamma(1-\alpha(k))} \int_{0}^{\infty} \sum_{\ell=1}^{\infty} \frac{(-1)^{\ell}}{\ell !} x^{-\alpha(k)-1+\ell} \mathbb{E}\left[\|X(t)\|^{2 \ell(k+1)} \mid \mathcal{F}_{s}\right] d x$

Thus, we can use the multilinear property of a process $(X(t))_{t \geq 0}$ to compute an integral of an infinite series of even moments to find any odd moment of $\|X(t)\|$. Whether above formula is useful depends of course on the particular situation and possible closed form alternatives.

We remark that the Ornstein-Uhlenbeck process driven by a Wiener process considered in Example 7 is Gaussian and if $B$ is a separable Hilbert space we may calculate $\|X(t)\|^{2}=\sum_{i=1}^{\infty}\left\langle X, e_{i}\right\rangle^{2}$, where $\left\{e_{i}\right\}_{i \in \mathbb{N}}$ is an orthonormal basis for $B$. Hence $\|X(t)\|^{2 k}=\sum_{i 1, \ldots, i k}\left\langle X, e_{i 1}\right\rangle^{2} \ldots\left\langle X, e_{i k}\right\rangle^{2}$. By the Isserlis-Wick theorem the $2 k$-mixed moments $\mathbb{E}\left[\left\langle X, e_{i 1}\right\rangle^{2} \ldots\left\langle X, e_{i k}\right\rangle^{2}\right]$ may be calculated explicitly from the covariance operator of $X(t)$. As above one may then calculated the odd moments from the even moments. Even in the Gaussian case however, it is not clear how to calculate conditional moments and above proposed procedure may be used.

In case the space $B$ is a Banach algebra, we may calculate $\mathbb{E}\left[X(t)^{k} \mid \mathcal{F}_{s}\right]$ by Lemma 14 for (generalized) polynomial processes. To the best of our knowledge calculations of moments of this form have not been investigated yet. 
4.2. Applications to commodity markets. A forward contract is a financial arrangement where the seller promises to deliver an underlying commodity (like for example oil, coffee, aluminium or power) at an agreed price at some future time point. Entering such a contract at time $t \geq 0$ where delivery takes place at time $t+x, x \geq 0$ in the future, we denote the agreed forward price by $f(t, x)$. It is known (see Benth and Krühner [4) that $t \mapsto f(t, \cdot)$ can be interpreted as a stochastic process with values in some Hilbert space of continuous functions on $\mathbb{R}_{+}$, solving (mildly) the stochastic partial differential equation (5) with $\mathcal{A}=\partial / \partial x$. This model is a special class of a more general stochastic partial differential equation dynamics, belonging to the Heath-Jarrow-Morton modelling paradigm (see e.g. Filipović [12, Geman [15], Carmona and Tehranchi [8] for more on this, including the case of forward rates in fixed-income markets).

Following Benth and Krühner [4, a natural state space of the forward price curves is the Filipović space (see Filipović [12]). The Filipović space $H_{w}$ is defined for an increasing, continuous function $w: \mathbb{R}_{+} \rightarrow[1, \infty)$ with $w(0)=1$ to be the set of functions

$$
H_{w}:=\left\{g \in A C\left(\mathbb{R}_{+}, \mathbb{R}\right): \int_{0}^{\infty} w(x) g^{\prime}(x)^{2} d x<\infty\right\},
$$

where $A C\left(\mathbb{R}_{+}, \mathbb{R}\right)$ denotes the set of absolutely continuous functions from $\mathbb{R}_{+}$to $\mathbb{R}$. The scalar product $\left\langle g_{1}, g_{2}\right\rangle:=g_{1}(0) g_{2}(0)+\int_{0}^{\infty} w(x) g_{1}^{\prime}(x) g_{2}^{\prime}(x) d x$ for $g_{1}, g_{2} \in H_{w}$ makes $H_{w}$ a separable Hilbert space with norm $\|g\|_{w}^{2}=|\langle g, g\rangle|$. As already observed in Benth and Krühner [4] assuming $w^{-1} \in L^{1}\left(\mathbb{R}_{+}\right)$, the pointwise multiplication defines an algebra on $H_{w}$ and with the new norm $|\cdot|_{w, c}:=c \mid \cdot{ }_{w}$, where $c=\sqrt{1+8\left(1+\int_{0}^{\infty} w^{-1}(x) d x\right)}$ the space $H_{w}$ is actually a commutative Banach algebra.

On the commutative Banach algebra $B=H_{w}$, we have that the shift operator $\mathcal{S}_{t} g:=g(\cdot+t)$ defines a $C_{0}$-semigroup being a homomorphism. Moreover, the generator of $\left(\mathcal{S}_{t}\right)_{t>0}$ is the derivative operator $\partial / \partial x$. Thus, in light of the discussion in Section 3 , the forward curve dynamics $(f(t, \cdot))_{t>0}$ is given by the stochastic convolution process (4), and recalling (20), will become a generalized polynomial process on $H_{w}$ with respect to $(f(s, \cdot))_{0 \leq s \leq t<\infty}$. In representation 200, we will have $X^{\perp}(s ; t):=\int_{s}^{t} \mathcal{S}_{t-u} d W(u)$ with $\mathcal{S}_{t}$ being the shift operator. In addition it is also a multilinear process with the same decomposition.

Let us give an application where the generalized polynomial property comes in handy. In commodity markets, options on forwards are popular risk management products. Let us consider a general payoff given by a measurable function $h: \mathbb{R} \rightarrow \mathbb{R}$ on the forward with delivery time $x$. At time $t$, the holder can exercise the option yielding a payment

$$
h(f(t, x)) .
$$

The most prominent example is $h(z)=\max (z-K, 0)$ for a standard call option. Let $\delta_{x}$ denote the evaluation map at $x \geq 0$. It is shown in Filipović [11 that $\delta_{x}$ is a bounded linear functional on $H_{w}$. Thus,

$$
h(f(t, x))=h\left(\delta_{x} f(t)\right),
$$

and the price of the option at time $s \leq t$ is given by

$$
P(s, t)=\mathbb{E}\left[h\left(\delta_{x} f(t)\right) \mid \mathcal{F}_{s}\right]
$$


assuming zero risk-free interest rate (see Benth et al. [6]). Assume now that there exists a polynomial representation of $h$,

$$
h(z)=\sum_{i=0}^{\infty} h_{i} z^{i}
$$

For continuous functions such approximation is guaranteed by the Weierstrass Approximation Theorem by using Bernstein polynomials. Let further

$$
P(s, t)=\sum_{i=0}^{\infty} h_{i} \mathbb{E}\left[\left(\delta_{x} f(t)\right)^{i} \mid \mathcal{F}_{s}\right]
$$

As $\delta_{x}$ is a linear functional on $H_{w}$, using that $\delta_{x}(g \cdot h)=\delta_{x}(g) \delta_{x}(h)$ for any $g, h \in H_{w}$, we can apply a modified version of Lemma 20 to show that

$$
\delta_{x} \mathbb{E}\left[f(t)^{i} \mid \mathcal{F}_{s}\right]=\mathbb{E}\left[\delta_{x}\left(f(t)^{i}\right) \mid \mathcal{F}_{s}\right]=\mathbb{E}\left[\left(\delta_{x} f(t)\right)^{i} \mid \mathcal{F}_{s}\right]
$$

It follows from 20 that

$$
\begin{aligned}
P(s, t) & =\sum_{i=0}^{\infty} h_{i} \delta_{x} \mathbb{E}\left[f(t)^{i} \mid \mathcal{F}_{s}\right] \\
& =\sum_{i=0}^{\infty} h_{i} \delta_{x} \mathbb{E}\left[\left(X^{\perp}(s ; t)+X^{\|}(s ; t)\right)^{i} \mid \mathcal{F}_{s}\right] \\
& =\sum_{i=0}^{\infty} h_{i}\left(\sum_{k=0}^{i}\left(\begin{array}{l}
i \\
k
\end{array}\right) \delta_{x}\left(\mathbb{E}\left[\left(X^{\perp}(s ; t)\right)^{i-k}\right]\right) \delta_{x+t-s} f^{i}(s)\right)
\end{aligned}
$$

where we have used that $\delta_{x}(g \cdot h)=\delta_{x}(g) \delta_{x}(h)$ for any $g, h \in H_{w}$ and $\delta_{x} \mathcal{S}_{t}=\delta_{x+t}$ for every $x, t \geq 0$. Note that the quantities $\delta_{x+t-s} f^{i}(s)=f^{i}(s, x+t-s), 1 \leq i<\infty$ can be read off from the observed forward curve $f(s)$ at time $s$. Choosing a sufficiently large cutoff $n$, an approximation for the price of the option is given by

$$
P(s, t) \approx \sum_{i=0}^{n} h_{i}\left(\sum_{k=0}^{i}\left(\begin{array}{l}
i \\
k
\end{array}\right) \delta_{x}\left(\mathbb{E}\left[\left(X^{\perp}(s ; t)\right)^{i-k}\right]\right)\right) f^{i}(s, x+t-s) .
$$

In some cases $\mathbb{E}\left[\left(X^{\perp}(s ; t)\right)^{i-k}\right]$ may be calculated explicitly as for example if $(X(t))_{t \geq 0}$ is the Ornstein-Uhlenbeck process studied in Example 7. In other cases the calculation of $\mathbb{E}\left[\left(X^{\perp}(s ; t)\right)^{i-k}\right]$ requires numerical techniques. However, these quantities need to be calculated only once in order to evaluate a whole trading book with options maturing at the same time.

A similar representation can be found if the option holder can exercise the option to receive a payment

$$
h\left(f\left(t, x_{1}\right), f\left(t, x_{2}\right), \ldots, f\left(t, x_{n}\right)\right)
$$

with delivery times $0 \leq x_{1}<x_{2}<\cdots<x_{n}$ and $h: \mathbb{R}^{n} \rightarrow \mathbb{R}$ a measurable function. This generalization covers the important example of a spread option on two forwards with different delivery times (calendar spread option) in which case $n=2$ and $h\left(z_{1}, z_{2}\right)=\max \left(z_{1}-z_{2}, 0\right)$. For these more complicated options one has to use the fact that $(f(t, \cdot))_{t \geq 0}$ is a multilinear process with respect to $\left(X^{\|}(s ; t)\right)_{0 \leq s \leq t<\infty}$.

Remark 19. Recall the definition of the process $(X(t))_{t \geq 0}$, which was the basis for the previous example, based on the stochastic partial differential equation (5). To 
allow for a wider range of applications, it would be interesting to analyse processes $(X(t))_{t \geq 0}$ given as the mild solution of the stochastic partial differential equation

$$
d X(t)=\mathcal{A} X(t)+a(t, X(t)) d t+b(t, X(t)) d W(t)
$$

where $\mathcal{A}$ is again a (densely defined) generator of a $C_{0}$-semigroup $\left(\mathcal{S}_{t}\right)_{t \geq 0}$ and $a$ : $\mathbb{R}_{+} \times B \rightarrow B, b: \mathbb{R}_{+} \times B \rightarrow L(\widetilde{B}, B)$. Here $\widetilde{B}$ is the noise space (possibly equal to $B)$ and $(W(t))_{t \geq 0}$ is a Wiener or Lévy process defined on $\widetilde{B}$. Such equation has under appropriate conditions a mild solution (see for instance Theorem 4.5. (1) in Tappe [19]) given by

$$
X(t)=\mathcal{S}_{t} X(0)+\int_{0}^{t} \mathcal{S}_{t-s} a(s, X(s)) d s+\int_{0}^{t} \mathcal{S}_{t-s} b(s, X(s)) d W(s) .
$$

An interesting question for future research is then to find out if there exists a family $(X(s ; t))_{0 \leq s \leq t<\infty}$ of $B$-valued random variables, such that $X(s ; t)$ is strongly $\mathcal{F}_{s^{-}}$ measurable and such that $(X(t))_{t \geq 0}$ is a multilinear or (generalized) polynomial process with respect to the family $(X(s ; t))_{0 \leq s \leq t<\infty}$.

4.3. Some other choices of Banach spaces. A canonical example of a separable Banach space is the space $C([0,1])$ of real-valued continuous functions $f:[0,1] \rightarrow \mathbb{R}$ equipped with pointwise product and uniform norm $|f|_{\infty}:=\sup _{x \in[0,1]}|f(x)|$. This is also a commutative Banach algebra, and we notice that it is the path space of Brownian motion.

Another classical separable Banach space is $L^{p}\left(\mathbb{R}^{d}\right)$, the space of $p$-integrable functions on $\mathbb{R}^{d}$ for $p, d \in \mathbb{N}$. As is well-known, $L^{2}\left(\mathbb{R}^{d}\right)$ is a Hilbert space and also possible state-space for Gaussian random fields. One can define a multiplication for $f, g \in L^{1}\left(\mathbb{R}^{d}\right)$ by the convolution product, i.e.

$$
f * g(x)=\int_{\mathbb{R}^{d}} f(y-x) g(y) d y .
$$

This turns $L^{1}\left(\mathbb{R}^{d}\right)$ into a commutative Banach algebra. A possible application could be stochastic processes taking values in $L^{1}(\mathbb{R})$ being probability densities, e.g. being non-negative integrable functions with unit mass.

Another classical Banach algebra is the space of bounded linear operators $B=$ $L(C)$ on the Banach space $C$, equipped with the operator norm. The space $B$ forms a non-commutative Banach algebra under the standard operator product. If $C$ is separable Hilbert space, one can consider the subspace of Hilbert-Schmidt operators $L_{\mathrm{HS}}(C)$, which becomes a separable Hilbert space and in addition a Banach algebra. In Benth, Rüdiger and Süss [5] and Benth and Simonsen [7] positive-definite HilbertSchmidt-valued Ornstein-Uhlenbeck processes have been defined and studied in the context of stochastic volatility models in infinite dimensions. These volatility models become multilinear processes.

Let $(E, \mathcal{E})$ be a measurable space, and denote by $\mathbb{M}(E)$ the space of all finite signed measures. Equip $\mathbb{M}(E)$ with the total variation norm, $\|\nu\|_{\mathrm{TV}}:=|\nu|(E)$ for $\nu \in \mathbb{M}(E)$. It is known that $\left(\mathbb{M}(E),\|\cdot\|_{\mathrm{TV}}\right)$ is a Banach space. Define the convolution product of measures as

$$
\nu * \mu(A)=\int_{E \times E} \mathbf{1}_{A}(x+y) \nu(d x) \mu(d y)=\int_{E} \nu(A-y) \mu(d y)
$$

for $\nu, \mu \in \mathbb{M}(E)$ and $A \in \mathcal{E}$. Since $\|\nu * \mu\|_{\mathrm{TV}} \leq\|\nu\|_{\mathrm{TV}}\|\mu\|_{\mathrm{TV}},\left(\mathbb{M}(E),\|\cdot\|_{\mathrm{TV}}, *\right)$ is a Banach algebra which obviously is commutative. A polynomial $p_{k} \in \operatorname{Pol}_{k}(\mathbb{M}(E))$ 
will be of the form $p_{k}(\mu)=\sum_{n=0}^{k} \nu_{n} * \mu^{* n}$ for $\left(\nu_{n}\right)_{n=0}^{k} \subset \mathbb{M}(E)$. These polynomials are built up from the monomials $\mu^{* n}$. Cuchiero, Larsson and Svaluto-Ferro [10] define polynomial processes on $\mathbb{M}(E)$ introducing monomials as follows: Let $g$ : $E^{k} \rightarrow \mathbb{R}$ be a continuous symmetric function. A monomial of degree $k \in \mathbb{N}$ is defined as

$$
\mathbb{M}(E) \ni \nu \mapsto\left\langle g, \nu^{k}\right\rangle:=\int_{E^{k}} g\left(x_{1}, \ldots, x_{k}\right) \nu\left(d x_{1}\right) \cdots \nu\left(d x_{k}\right)
$$

We notice that for any $A \in \mathcal{E}$, we have that

$$
\nu^{* k}(A)=\int_{E^{k}} \mathbb{I}_{A}\left(x_{1}+\cdots+x_{k}\right) \nu\left(d x_{1}\right) \cdots \nu\left(d x_{k}\right)=\left\langle\mathbb{I}_{A}\left(x_{1}+\cdots+x_{k}\right), \nu^{k}\right\rangle .
$$

Although the function $\left(x_{1}, \ldots, x_{k}\right) \mapsto \mathbb{I}_{A}\left(x_{1}+\cdots+x_{k}\right)$ is obviously not continuous, it is a bounded measurable symmetric function which is linking our definition of polynomial processes on $\mathbb{M}(E)$ to the one of Cuchiero, Larsson and SvalutoFerro [10].

\section{REFERENCES}

[1] D. Ackerer, D. Filipović and S. Pulido (2018). The Jacobi stochastic volatility model. Finance Stoch. 22: 667-700.

[2] D. Applebaum (2004). Lévy Processes and Stochastic Calculus, Cambridge University Press

[3] D. Applebaum (2007). Lévy processes and stochastic integrals in Banach spaces. Prob. Math. Stat. 27: $75-88$.

[4] F. E. Benth and P. Krühner (2014). Representation of infinite-dimensional forward price models in commodity markets. Commun. Math. Stat. 2:47-106

[5] F. E. Benth, B. Rüdiger and A. Süss (2018). Ornstein-Uhlenbeck processes in Hilbert space with non-Gaussian stochastic volatility. Stoch. Proc. Applic., 128: 461-486.

[6] F. E. Benth, J. Šaltytė Benth, and S. Koekebakker (2008). Stochastic Modelling of Electricity and Related Markets, World Scientific, Singapore.

[7] F. E. Benth and I. C. Simonsen (2018). The Heston stochastic volatility model in Hilbert space. Stoch. Analysis Appl. 36: 733-750.

[8] R. Carmona, and M. Tehranchi (2006). Interest Rate Models: an Infinite Dimensional Stochastic Analysis Perspective, Springer Verlag

[9] C. Cuchiero, M. Keller-Ressel and J. Teichmann (2012). Polynomial processes and their applications to mathematical finance. Finance Stoch. 16:711-740

[10] C. Cuchiero, M. Larsson and S. Svaluto-Ferro (2018). Measure valued polynomial diffusions. Working paper.

[11] N. Dinculeanu (2000). Vector Integration and Stochastic Integration in Banach Spaces. John Wiley \& Sons.

[12] D. Filipović (2001). Consistency Problems for Heath-Jarrow-Morton Interest Rate Models. Lecture Notes in Mathematics, vol. 1760. Springer, Berlin.

[13] D. Filipović and M. Larsson (2016). Polynomial diffusions and applications in finance. Finance Stoch. 4: 931-972

[14] J.L. Forman and M. Sørensen (2008). The Pearson diffusions: a class of statistically tractable diffusion processes. Scand. J. Stat. 35: 438-465

[15] H. Geman (2005). Commodities and Commodity Derivatives. John Wiley \& Sons, Chichester. [16] J. van Neerven (2010). Stochastic Evolution Equations, ISEM Lecture Notes 2007/8. Downloaded from http://fa.its.tudelft.nl/ neerven/publications/notes/ISEM.pdf in May 2018.

[17] S. Peszat and J. Zabczyk (2007). Stochastic Partial Differential Equations with Lévy Noise.. Cambridge University Press

[18] G. Da Prato and J. Zabczyk (2014). Stochastic Equations in Infinite Dimensions. Cambridge University Press

[19] S. Tappe (2012). Some refinements of existence results for spdes driven by wiener processes and poisson random measures. International Journal of Stochastic Analysis 
[20] J. Walsh (1986), An introduction to stochastic partial differential equations. In R. Carmona, H. Kesten \& J. Walsh (eds.), Lecture Notes in Mathematics, 1180, Ecole d'Eté de Probabilités de Saint-Flour XIV (1984), Springer Verlag.

\section{Appendix A. Some AUXiliary RESUlts on CONDITIONAL EXPECTATION IN BANACH SPACES}

Let $B$ be a Banach space over a field $\mathbb{F}$, which can be either $\mathbb{R}$ or $\mathbb{C}$. We denote the norm by $\|\cdot\|$ and by $\mathcal{B}(B)$ the Borel $\sigma$-algebra of $B$. Further, $L(B)$ denotes the space of bounded linear operators on $B$. Let $(\Omega, \mathcal{F}, \mathbb{P})$ be a probability space equipped with a filtration $\left(\mathcal{F}_{t}\right)_{t \geq 0}$. Following the usual terminology (see e.g. Def. 1.4 in van Neerven [16]), a $B$-valued random variable $X$ is a mapping from $\Omega$ into $B$ which is strongly measurable, that is, there exists a sequence of simple random variables $X_{n}:=\sum_{i=1}^{n} \mathbb{I}_{F_{i}} x_{i}$ where $F_{i} \in \mathcal{F}, x_{i} \in B, i=1, \ldots, n, n \in \mathbb{N}$ such that $X_{n} \rightarrow X$ in $B$ pointwise when $n \rightarrow \infty$. Here, we use the notation $\mathbb{I}_{F}: \Omega \rightarrow\{0,1\}$ as the indicator function on a set $F \in \mathcal{F}$. If $B$ is separable, then strong measurability is equivalent to measurability in the sense that $X^{-1}(A) \in \mathcal{F}$ for any $A \in \mathcal{B}(B)$. As a consequence of the approximation $X_{n} \rightarrow X$ a random variable $X$ takes values in the closed separable subspace $B_{c}:=\overline{\text { span } \cup_{n \in \mathbb{N}} \operatorname{ran}\left(X_{n}\right)}$, the closure of the subspace spanned by the ranges of the $X_{n}$.

In van Neerven [16, a mapping $X: \Omega \rightarrow B$ is said to be strongly $\mathbb{P}$-measurable if there exists a sequence of simple random variables $X_{n}$ such that $X_{n} \rightarrow X$ in $B$ $\mathbb{P}-$ a.s as $n \rightarrow \infty$. However, in view of Prop. 1.10 in van Neerven [16], there exists a version $\tilde{X}$ which is strongly measurable of any strongly $\mathbb{P}$-measurable random variable $X$, and vice versa. Thus, in our analysis we will always choose the strongly measurable version of a random variable, and therefore stick to the notion of strongly measurable throughout.

A random variable $X$ where $\mathbb{E}[\|X\|]<\infty$ is said to be Bochner integrable with respect to $\mathbb{P}$, and we define $\mathbb{E}[X]$ to be the Bochner integral (see e.g. Ch. $1 \S 1(\mathrm{~J})$ of Dinculeanu [11])

$$
\mathbb{E}[X]:=\int_{\Omega} X d \mathbb{P}
$$

Moreover, $\mathbb{E}[X] \in B$ and $\|\mathbb{E}[X]\| \leq \mathbb{E}[\|X\|]$. Given a $\sigma$-algebra $\mathcal{G} \subset \mathcal{F}$ and a Bochner integrable $B$-valued random variable, we define the conditional expectation $\mathbb{E}[X \mid \mathcal{G}]$ as the strongly $\mathcal{G}$-measurable random variable satisfying

$$
\int_{G} \mathbb{E}[X \mid \mathcal{G}] d \mathbb{P}=\int_{G} X d \mathbb{P}
$$

for all $G \in \mathcal{G}$ (see Def. 38 in Ch. $1 \S 2$ of Dinculeanu 11]). Thm. 50 and Prop. 37 in Ch. $1 \S 2$ of Dinculeanu [11 show that the conditional expectation exists and is unique $\mathbb{P}-$ a.s..

The next result shows that (conditional) expectation commutes with bounded linear operators:

Lemma 20. Suppose that $X$ is a B-valued random variable which is Bochner integrable and $\mathcal{L} \in L(B)$. Then $\mathcal{L} X$ is a B-valued random variable which is Bochner integrable, $\mathbb{E}[\mathcal{L} X]=\mathcal{L} \mathbb{E}[X]$ and

$$
\mathbb{E}[\mathcal{L} X \mid \mathcal{G}]=\mathcal{L} \mathbb{E}[X \mid \mathcal{G}]
$$

for any $\mathcal{G} \subset \mathcal{F}$. 
Proof. Since there exists a closed separable subspace $B_{c} \subseteq B$ with $\mathbb{P}\left[X \in B_{c}\right]=1$, we know that also the range of $\mathcal{L}$ is separable. Now, the statement is given in Peszat and Zabczyk [17, Proposition 3.15(ii)].

As the following Lemma shows, if $f: B \rightarrow B$ is continuous, then $f(X)$ is strongly measurable whenever $X$ is. This is stronger than the first claim in the Lemma 20 above. Indeed, the more general result holds:

Lemma 21. If $f: B_{1} \rightarrow B_{2}$ is a continuous map between two Banach spaces $B_{1}$ and $B_{2}$, then $f(X)$ is a strongly measurable $B_{2}$-valued random variable for any strongly measurable $B_{1}$-valued random variable $X$.

Proof. For any sequence of simple random variables $X_{n}$ converging to $X$, we have that $f\left(X_{n}\right)$ is converging to $f(X)$ by continuity. If $X_{n}=\sum_{i=1}^{n} \mathbb{I}_{F_{i}} x_{i}$ for disjoint sets $F_{i} \in \mathcal{F}$ (we can always do this by redefining the sum), we find $f\left(X_{n}\right)=$ $\sum_{i=1}^{n} \mathbb{I}_{F_{i}} f\left(x_{i}\right)$, which shows that $\left(f\left(X_{n}\right)\right)_{n \in \mathbb{N}}$ is a sequence of simple random variables converging pointwise to $f(X)$. Hence, $f(X)$ is strongly measurable.

Choosing $B=B_{1}$ and $B_{2}=\mathbb{R}$, we find that $\|X\|$ is a measurable real-valued random variable when $X$ is a $B$-valued strongly measurable random variable. This is true since $x \mapsto\|x\|$ is a continuous map, as the triangle inequality shows that $\|x\| \leq\|x-y\|+\|y\|$ and $\|y\| \leq\|x-y\|+\|x\|$, and therefore $|\|x\|-\|y\|| \leq\|x-y\|$.

Let us focus on the concept of independence for $B$-valued random variables. We recall that $X$ is independent of a $\sigma$-algebra $\mathcal{G} \subset \mathcal{F}$ if the two sets $X^{-1}(A)$ and $G \in \mathcal{G}$ are independent for all $A \in \mathcal{B}(B)$ and $G \in \mathcal{G}$. If $f: B \rightarrow B$ is a measurable map, it follows that $f(X)$ is independent of $\mathcal{G}$ whenever $X$ is independent of $\mathcal{G}$. This is so because for any $A \in \mathcal{B}(B),(f(X))^{-1}(A)=X^{-1}\left(f^{-1}(A)\right)$ and $f^{-1}(A) \in \mathcal{B}(B)$ as $f$ is measurable. Moreover, if in addition $f$ is continuous, we see from Lemma 21 that $f(X)$ is a strongly measurable random variable being independent of $\mathcal{G}$. As a particular case, assume that $B$ is a Banach algebra, that is, $B$ is equipped with a multiplication operator $\cdot: B \times B \rightarrow B$ such that $(B,+, \cdot)$ is an associative $\mathbb{F}$-algebra and $\|x \cdot y\| \leq\|x\|\|y\|$ for any $x, y \in B$. Consider the map $f: B \ni x \mapsto x^{2} \in B$. By the norm property in a Banach algebra,

$$
\left\|x^{2}-y^{2}\right\|=\|x(x-y)+(x-y) y\| \leq(\|x\|+\|y\|)\|x-y\|,
$$

it follows that $f$ is continuous. Thus, we see that $X^{2}$ is independent of $\mathcal{G}$ whenever $X$ is independent of $\mathcal{G}$. By induction, we have that $X^{k}$ is independent of $\mathcal{G}$ whenever $X$ is independent of $\mathcal{G}$ for any $k \in \mathbb{N}$.

We have the following result on conditional expectation of independent random variables:

Lemma 22. If $X$ is a B-valued Bochner-integrable random variable which is independent of the $\sigma$-algebra $\mathcal{G} \subset \mathcal{F}$, then

$$
\mathbb{E}[X \mid \mathcal{G}]=\mathbb{E}[X] .
$$

Proof. Since $X$ is Bochner-integrable there is a separable closed subspace $B_{c} \subseteq B$ such that $\mathbb{P}\left[X \in B_{c}\right]=1$. Consequently, we may assume that $B$ is separable. The statement is given in Peszat and Zabczyk [17, Proposition 3.15(v)].

In our analysis, a "freezing property" of conditional expectation is important. To this end, we equip the product space $B \times B$ of the Banach space $B$ with the 
max-norm, i.e., for any $x=\left(x_{1}, x_{2}\right) \in B \times B,\|x\|_{2}:=\max _{i=1,2}\left\|x_{i}\right\|$. Then, $B \times B$ is a Banach space again.

Proposition 23. Let $\mathcal{G} \subset \mathcal{F}$ be a $\sigma$-algebra and $X$ a $B$-valued random variable independent of $\mathcal{G}$. Let further $Y$ be a B-valued random variable which is strongly $\mathcal{G}$-measurable and $f: B \times B \rightarrow B$ continuous. Assume $f(X, y)$ is Bochner integrable for every $y \in B$ and $y \mapsto \mathbb{E}[f(X, y)]$ is continuous, and moreover that there exist positive $\mathbb{R}$-valued random variables $Z, \widetilde{Z}$ with $\mathbb{E}[Z]<\infty, \mathbb{E}[\widetilde{Z}]<\infty$ and

$$
\begin{array}{r}
\left\|\mathbb{E}[f(X, y)]_{y=\tilde{Y}}\right\| \leq Z \\
\|f(X, \tilde{Y})\| \leq \tilde{Z},
\end{array}
$$

for every $B$-valued random variable $\tilde{Y}$ such that $\|\widetilde{Y}\| \leq\|Y\|$ a.s., then

$$
\mathbb{E}[f(X, Y) \mid \mathcal{G}]=\mathbb{E}[f(X, y)]_{y=Y} .
$$

Proof. First, by Lemma 21, we note that continuity of $f$ implies that $f(X, Y)$ and $f(X, y)$ are strongly measurable for any $y \in B$ (using $B_{2}=B$ and $B_{1}=B \times B$ or $\left.B_{2}=B\right)$. Choosing $\widetilde{Y}=Y$ in $(30)$, we find that $\mathbb{E}[\|f(X, Y)\|] \leq \mathbb{E}[\widetilde{Z}]<\infty$, and therefore $f(X, Y)$ is Bochner integrable.

We need to show that $\mathbb{E}\left[\mathbb{I}_{G} f(X, Y)\right]=\mathbb{E}\left[\mathbb{I}_{G} \mathbb{E}[f(X, y)]_{y=Y}\right]$ for any $G \in \mathcal{G}$. For this, let $G \in \mathcal{G}$ and recall that since $Y$ is strongly $\mathcal{G}$-measurable, there exists a sequence of $\mathcal{G}$-simple random variables $Y_{n}=\sum_{i=1}^{n} y_{i} \mathbb{I}_{A_{i}}$ with $y_{i} \in B$ and $A_{i} \in \mathcal{G}$ such that $Y_{n} \rightarrow Y$ pointwise. Moreover, by Thm. 6 in Ch. $1 \S 1 \mathrm{C}$ of Dinculeanu [1] we can choose $Y_{n}$ such that $\left\|Y_{n}\right\| \leq\|Y\|$. We also notice that the we can select the sets $A_{1}, \ldots, A_{n}$ to be disjoint, as we do. We see that

$$
f\left(X, Y_{n}\right)=\sum_{i=1}^{n} \mathbb{I}_{A_{i}} f\left(X, y_{i}\right)
$$

and by assumption $(30)$, we calculate

$$
\begin{aligned}
\infty>\mathbb{E}\left[\left\|f\left(X, Y_{n}\right)\right\|\right] & =\mathbb{E}\left[\sum_{i=1}^{n} \mathbb{I}_{A_{i}}\left\|f\left(X, y_{i}\right)\right\|\right] \\
& =\sum_{i=1}^{n} \mathbb{E}\left[\mathbb{I}_{A_{i}}\left\|f\left(X, y_{i}\right)\right\|\right] \\
& =\sum_{i=1}^{n} \mathbb{P}\left(A_{i}\right) \mathbb{E}\left[\left\|f\left(X, y_{i}\right)\right\|\right] .
\end{aligned}
$$

In the last equality we used the fact that $\left\|f\left(X, y_{i}\right)\right\|$ is independent on $\mathcal{G}$, as $f$ and $\|\cdot\|$ are continuous functions and $X$ is independent of $\mathcal{G}$ by assumption. In particular, this shows that $\mathbb{E}\left[\left\|f\left(X, y_{i}\right)\right\|\right]<\infty$, and hence $f\left(X, y_{i}\right)$ is Bochner integrable. Therefore, by Lemma 22 it follows that

$$
\mathbb{E}\left[f\left(X, y_{i}\right)\right]=\mathbb{E}\left[f\left(X, y_{i}\right) \mid \mathcal{G}\right]
$$

On the other hand,

$$
\mathbb{E}[f(X, y)]_{y=Y_{n}}=\sum_{i=1}^{n} \mathbb{I}_{A_{i}} \mathbb{E}\left[f\left(X, y_{i}\right)\right]
$$


Hence, $\mathbb{E}[f(X, y)]_{y=Y_{n}}$ is strongly $\mathcal{G}$-measurable and Bochner integrable since, from norm inequality of Bochner integrals and assumption (30)

$$
\begin{aligned}
\mathbb{E}\left[\left\|\mathbb{E}[f(X, y)]_{y=Y_{n}}\right\|\right] & =\mathbb{E}\left[\sum_{i=1}^{n} \mathbb{I}_{A_{i}}\left\|\mathbb{E}\left[f\left(X, y_{i}\right)\right]\right\|\right] \\
& =\sum_{i=1}^{n} \mathbb{P}\left(A_{i}\right)\left\|\mathbb{E}\left[f\left(X, y_{i}\right)\right]\right\| \\
& \leq \sum_{i=1}^{n} \mathbb{P}\left(A_{i}\right) \mathbb{E}\left[\left\|f\left(X, y_{i}\right)\right\|\right] \\
& =\mathbb{E}\left[\left\|f\left(X, Y_{n}\right)\right\|\right]<\infty
\end{aligned}
$$

Thus, we calculate

$$
\begin{aligned}
\mathbb{E}\left[\mathbb{I}_{G} \mathbb{E}[f(X, y)]_{y=Y_{n}}\right] & =\mathbb{E}\left[\sum_{i=1}^{n} \mathbb{I}_{G} \mathbb{I}_{A_{i}} \mathbb{E}\left[f\left(X, y_{i}\right)\right]\right] \\
& =\sum_{i=1}^{n} \mathbb{E}\left[\mathbb{I}_{G \cap A_{i}} \mathbb{E}\left[f\left(X, y_{i}\right)\right]\right] \\
& =\sum_{i=1}^{n} \mathbb{E}\left[\mathbb{I}_{G \cap A_{i}} f\left(X, y_{i}\right)\right] \\
& =\mathbb{E}\left[\mathbb{I}_{G} f\left(X, Y_{n}\right)\right],
\end{aligned}
$$

where the third equality uses (31) and the defining properties of conditional expectation. To see that in fact $\mathbb{E}\left[\mathbb{I}_{G} \mathbb{E}[f(X, y)]_{y=Y}\right]=\mathbb{E}\left[\mathbb{I}_{G} f(X, Y)\right]$ we need to show that $\lim _{n \rightarrow \infty} \mathbb{E}\left[\mathbb{I}_{G} \mathbb{E}[f(X, y)]_{y=Y_{n}}\right]=\mathbb{E}\left[\mathbb{I}_{G} \mathbb{E}[f(X, y)]_{y=Y}\right]$ and $\lim _{n \rightarrow \infty} \mathbb{E}\left[\mathbb{I}_{G} f\left(X, Y_{n}\right)\right]=$ $\mathbb{E}\left[\mathbb{I}_{G} f(X, Y)\right]$. For this note that since $f$ is continuous, $\mathbb{I}_{G} f\left(X, Y_{n}\right) \rightarrow \mathbb{I}_{G} f(X, Y)$ when $n \rightarrow \infty$. As $\left\|Y_{n}\right\| \leq\|Y\|$, we have

$$
\left\|\mathbb{I}_{G} f\left(X, Y_{n}\right)\right\| \leq \mathbb{I}_{G}\left\|f\left(X, Y_{n}\right)\right\| \leq \tilde{Z}
$$

and thus from dominated convergence it follows that

$$
\lim _{n \rightarrow \infty} \mathbb{E}\left[\mathbb{I}_{G} f\left(X, Y_{n}\right)\right]=\mathbb{E}\left[\mathbb{I}_{G} f(X, Y)\right]
$$

For the other limit, we have from the continuity assumption on $y \mapsto \mathbb{E}[f(X, y)]$ that $\mathbb{I}_{G} \mathbb{E}[f(X, y)]_{y=Y_{n}} \rightarrow \mathbb{I}_{G} \mathbb{E}[f(X, y)]_{y=Y}$ pointwise in $B$. Furthermore, by assumption 29

$$
\left\|\mathbb{I}_{G} \mathbb{E}[f(X, y)]_{y=Y_{n}}\right\| \leq \mathbb{I}_{G}\left\|\mathbb{E}[f(X, y)]_{y=Y_{n}}\right\| \leq Z
$$

Then, by dominated convergence, we obtain

$$
\lim _{n \rightarrow \infty} \mathbb{E}\left[\mathbb{I}_{G} \mathbb{E}[f(X, y)]_{y=Y_{n}}\right]=\mathbb{E}\left[\mathbb{I}_{G} \mathbb{E}[f(X, y)]_{y=Y}\right],
$$

and the proposition follows.

Remark that condition 29 is only used once, to obtain a uniform bound on $\left\|\mathbb{E}[f(X, y)]_{y=Y_{n}}\right\|$. Further, the continuity assumption on the function $y \mapsto \mathbb{E}[f(X, y)]$ is only used to have pointwise convergence. Both are used only in connection with concluding the final limit in the proof above.

In the remainder of this appendix we will focus on the case when $B$ is a Banach algebra, and in particular show the following fundamental property for conditional 
expectation: Let $\mathcal{G} \subset \mathcal{F}$ be a $\sigma$-algebra and $Y$ a $\mathcal{G}$-strongly measurable $B$-valued random variable, then

$$
\mathbb{E}[Y X \mid \mathcal{G}]=Y \mathbb{E}[X \mid \mathcal{G}], \mathbb{E}[X Y \mid \mathcal{G}]=\mathbb{E}[X \mid \mathcal{G}] Y
$$

where $X$ is a $B$-valued random variable, with $X, Y X$ and $X Y$ such that the conditional expectations are well-defined.

First, we show a Lemma which will become convenient:

Lemma 24. Let $(S, \Sigma, \mu)$ be a measure space and $B$ a Banach algebra. Suppose $F:(S, \Sigma, \mu) \rightarrow B$ is $\mu$-integrable (that is, Bochner integrable with respect to $\mu$ ) and $g \in B$. Then $g F$ and $F g$ are $\mu$-integrable, and

$$
\int_{S} g F(s) \mu(d s)=g \int_{S} F(s) \mu(d s) \quad \int_{S} F(s) g \mu(d s)=\int_{S} F(s) \mu(d s) g .
$$

Proof. Define the continuous linear maps

$$
\begin{gathered}
\mathcal{L}_{g}: B \rightarrow B, b \mapsto g b, \\
\mathcal{R}_{g}: B \rightarrow B, b \mapsto b g .
\end{gathered}
$$

Then we find

$$
\int_{S} F(s) g \mu(d s)=\int_{S} \mathcal{R}_{g} F(s) \mu(d s)=\mathcal{R}_{g}\left(\int_{S} \mathcal{F}(s) \mu(d s)\right)=\int_{S} \mathcal{F}(s) \mu(d s) g .
$$

Similar with $\mathcal{L}_{g}$.

The next Lemma shows that measurability is preserved under the product operation in the Banach algebra:

Lemma 25. Let $\mathcal{G} \subset \mathcal{F}$ be a $\sigma$-algebra, and suppose that $Y$ and $Z$ are two strongly $\mathcal{G}$-measurable $B$-valued random variables and that $B$ is a Banach algebra. Then $Y Z$ and $Z Y$ are strongly $\mathcal{G}$-measurable $B$-valued random variables.

Proof. The pair $(Y, Z)$ is strongly $B \times B$-measurable and the multiplication $\eta$ on $B$ is a continuous map from $B \times B$ to $B$. Thus, Lemma 21 yields that $Y Z=\eta(Y, Z)$ is strongly $\mathcal{G}$-measurable.

We come to our final result of this appendix:

Proposition 26. Let $B$ be a Banach algebra and $\mathcal{G} \subset \mathcal{F}$ a $\sigma$-algebra. Assume that $X$ and $Y$ are two $B$-valued random variables where $Y$ is strongly $\mathcal{G}$-measurable, $X$ is Bochner-integrable and $\|X\|\|Y\|$ is $\mathbb{P}$-integrable. Then

$$
\mathbb{E}[Y X \mid \mathcal{G}]=Y \mathbb{E}[X \mid \mathcal{G}], \quad \mathbb{E}[X Y \mid \mathcal{G}]=\mathbb{E}[X \mid \mathcal{G}] Y .
$$

Proof. First, $\|X Y\| \leq\|X\|\|Y\|$ and $\|Y X\| \leq\|Y\|\|X\|$, so both $X Y$ and $Y X$ are Bochner integrable, and moreover, the conditional expectations of $X Y$ and $Y X$ with respect to $\mathcal{G}$ are well-defined. By assumption, the conditional expectation of $X$ with respect to $\mathcal{G}$ is also well-defined.

By definition of the conditional expectation, $\mathbb{E}[X \mid \mathcal{G}]$ is strongly $\mathcal{G}$-measurable, and thus by Lemma $25, Y \mathbb{E}[X \mid \mathcal{G}]$ is $\mathcal{G}$-strongly measurable. Let $Y_{n}=\sum_{i=1}^{n} y_{i} \mathbb{I}_{A_{i}}$ with $y_{i} \in B$ and $A_{i} \in \mathcal{G}$ for $i=1, \ldots, n$ be a sequence of $\mathcal{G}$-simple random variables 
such that $Y_{n} \rightarrow Y$ pointwise and by Thm. 6 in Ch. $1 \S 1 \mathrm{C}$ of Dinculeanu [11, $\left\|Y_{n}\right\| \leq\|Y\|$. Let $G \in \mathcal{G}$ be an arbitrary set. We find

$$
\begin{aligned}
\mathbb{E}\left[\mathbb{I}_{G} Y_{n} X\right] & =\sum_{i=1}^{n} \mathbb{E}\left[\mathbb{I}_{G} \mathbb{I}_{A_{i}} y_{i} X\right] \\
& =\sum_{i=1}^{n} y_{i} \mathbb{E}\left[\mathbb{I}_{G \cap A_{i}} X\right] \\
& =\sum_{i=1}^{n} y_{i} \mathbb{E}\left[\mathbb{I}_{G \cap A_{i}} \mathbb{E}[X \mid \mathcal{G}]\right] \\
& =\sum_{i=1}^{n} \mathbb{E}\left[\mathbb{I}_{G} \mathbb{I}_{A_{i}} y_{i} \mathbb{E}[X \mid \mathcal{G}]\right] \\
& =\mathbb{E}\left[\mathbb{I}_{G} Y_{n} \mathbb{E}[X \mid \mathcal{G}]\right]
\end{aligned}
$$

In the second and fourth equalities we applied Lemma 24, and in the third the definition of the conditional expectation. Now,

$$
\left\|\mathbb{I}_{G} Y_{n} X\right\| \leq \mathbb{I}_{G}\left\|Y_{n}\right\|\|X\| \leq\|Y\|\|X\| \in L^{1}(P)
$$

by assumption. Thus, as $Y_{n} X \rightarrow Y X$, it follows by dominated convergence that $\mathbb{E}\left[\mathbb{I}_{G} Y_{n} X\right] \rightarrow \mathbb{E}\left[\mathbb{I}_{G} Y X\right]$. On the other hand,

$$
\left.\| \mathbb{I}_{G} Y_{n} \mathbb{E}[X \mid \mathcal{G}]\right]\left\|\leq \mathbb{I}_{G}\right\| Y_{n}\|\mathbb{E}[\|X\| \mid \mathcal{G}] \leq\| Y \| \mathbb{E}[\|X\| \mid \mathcal{G}] .
$$

a.s., by Jensen's inequality (Property 44 in Ch.1§2 H of Dinculeanu [11]). As $Y$ is strongly $\mathcal{G}$-measurable, it follows from Lemma 21 that $\|Y\|$ is a real-valued $\mathcal{G}$ measurable random variable. From the properties of conditional expectation for real-valued random variables

$$
\mathbb{E}[\|Y\| \mathbb{E}[\|X\| \mid \mathcal{G}]]=\mathbb{E}[\mathbb{E}[\|Y\|\|X\| \mid \mathcal{G}]]=\mathbb{E}[\|Y\|\|X\|]<\infty
$$

by assumption. Hence, $\|Y\| \mathbb{E}[\|X\| \mid \mathcal{G}]$ is $P$-integrable, and since obviously it holds pointwise that $\mathbb{I}_{G} Y_{n} \mathbb{E}[X \mid \mathcal{G}] \rightarrow \mathbb{I}_{G} Y \mathbb{E}[X \mid \mathcal{G}]$ we find by dominated convergence that $\mathbb{E}\left[\mathbb{I}_{G} Y_{n} \mathbb{E}[X \mid \mathcal{G}]\right] \rightarrow \mathbb{E}\left[\mathbb{I}_{G} Y \mathbb{E}[X \mid \mathcal{G}]\right]$. We can therefore conclude

$$
\mathbb{E}\left[\mathbb{I}_{G} Y \mathbb{E}[X \mid \mathcal{G}]\right]=\lim _{n \rightarrow \infty} \mathbb{E}\left[\mathbb{I}_{G} Y_{n} \mathbb{E}[X \mid \mathcal{G}]\right]=\lim _{n \rightarrow \infty} \mathbb{E}\left[\mathbb{I}_{G} Y_{n} X\right]=\mathbb{E}\left[\mathbb{I}_{G} Y X\right] .
$$

Hence, the first result of the Proposition is proven. The second part follows in the same manner.

Fred Espen Benth, University of Oslo, Department of Mathematics, P.O. Box 1053, Blindern, N-0316 Oslo, Norway

E-mail address: fredb@math.uio.no

Nils Detering, Department of Statistics and Applied Probability, CA 93106 Santa BARBARA, USA

E-mail address: detering@pstat.ucsb.edu

Paul Krühner, Institute for Financial and Actuarial Mathematics, The University of Liverpool, The Mathematical Sciences Building, Liverpool L69 7ZL, UK

E-mail address: paul.eisenberg@liverpool.ac.uk 